\title{
Phase Diagrams and Electromagnetic Propertiesof s-wave Superconductivity of the Extended Hubbard Model with the Attractive Pair-Hopping Interaction
}

\author{
W. R. Czart $^{1}$ iD
}

Received: 23 August 2018 / Accepted: 29 November 2018 / Published online: 26 February 2019

(C) The Author(s) 2019

\begin{abstract}
The characteristics of the extended Hubbard model with the pair-hopping interaction $J$, i.e. the Penson-Kolb-Hubbard model, are analysed for the case of attractive $J(J>0)$. The effects of on-site $U$ interaction on the properties of the swave superconducting state and mutual stability of the superconducting s-wave and magnetic orderings are examined. The electromagnetic and thermodynamic characteristics of the s-wave superconducting phase for arbitrary particle concentration $n$ and interactions are analysed. The system is evaluated for the case of nearest-neighbours hopping on $d$-dimensional hypercubic $d=2$ (SQ) and $d=3$ (SC) nonfrustrated lattices. For SQ lattice the effects of phase fluctuations on the properties of the superconducting state are discussed within the Kosterlitz-Thouless (KT) scenario. The KT critical temperatures $T_{K T}$ are calculated and compared to the $T_{c}$ obtained within the BCS-HFA. The Uemura type plots, $T_{K T}$ vs. $\lambda^{2}(0)$, are obtained and evaluated as a function of pairing strength and concentration. The results are compared with the case of repulsive $J(J$ $<0)$ pair-hopping interaction.
\end{abstract}

Keywords Superconductivity $\cdot$ Phase separation $\cdot$ Extended Hubbard model $\cdot$ Mean-field $\cdot$ s-wave $\cdot$ Superconductivity Attractive pair-hopping interaction

\section{Introduction}

In this work we are interested in the pairing in the real space characterized by small coherence lengths. Superconductivity with short coherence length is appropriate to a description of the high-temperature superconductivity in materials such as the cuprates, doped bismuthates, ironbased systems, Chevrel phases, organic metals, fullerenes, etc. (for a review, see e.g. Refs. [1, 2, 4-6] and references therein).

One of the models often used for phenomenological description of dynamics of tightly-bound charged pairs is the attractive Hubbard (AH) model. In the AH model the effective on-site attractive interaction $(U<0)$ between charge carriers is responsible for pairs creation. Other models used to study the physics of pairing in the real

W. R. Czart

czart@amu.edu.pl

1 Faculty of Physics, Adam Mickiewicz University in Poznań, Umultowska 8561-614, Poznań, Poland space include the pair-hopping interaction $J[8,9,11]$. This interaction between the fermion pairs located at different sites (intersite charge exchange) can drive pairs forming and condensation.

In the present paper we analyse the Penson-KolbHubbard (PKH) model which includes both the on-site term $(U)$ as well as the pair hopping interaction $J$. This is conceptually a simple effective model for studying correlations, electron orderings and superconductivity in narrow band systems with almost unretarded short-range pairing [6, 9, 11-14, 16-19, 21, 23]. The model Hamiltonian is as follows:

$$
\begin{aligned}
H= & -\sum_{i j \sigma} t_{i j}\left(c_{i \sigma}^{+} c_{j \sigma}+\text { h.c. }\right)-\sum_{i \sigma} \mu n_{i \sigma} \\
& +U \sum_{i} n_{i \uparrow} n_{i \downarrow} \\
& -\frac{1}{2} \sum_{i j} J_{i j}\left(c_{i \uparrow}^{+} c_{i \downarrow}^{+} c_{\mathrm{j} \downarrow} c_{j \uparrow}+\text { h.c. }\right),
\end{aligned}
$$

where $t_{i j}$ are the single electron hopping integrals, $U$ is the on-site density-density interaction, $J_{i j}$ is the intersite charge 
exchange interaction between pairs, the chemical potential is represented by $\mu, n_{i \sigma}=c_{i \sigma}^{+} c_{i \sigma}$, and

$n=\frac{1}{N} \sum_{i \sigma}\left\langle n_{i \sigma}\right\rangle$.

In the model $J$ term competes with $U$ interaction. When $U$ $=0$ Hamiltonian (1) transforms to the Penson-Kolb (PK) model [8, 12, 14, 17, 25-29], whereas for $J=0$ and $U<0$ one gets the Hamiltonian of the AH model [1, 30, 31, 33].

So far, the PK and PKH models have been investigated only in a few limits [2, 9, 11, 13, 35-37]. The main efforts concerned characteristics at the ground state $(T=0)$ at halffilling $(n=1)$ in one dimension $(d=1)[9,11,35]$. At $T=0$, for both signs of $J$ and $U$, diagrams of the PKH model on $d=1$ and $d=\infty$ hypercubic lattices have been evaluated within the broken symmetry Hartree-Fock approximation (HFA) and by the slave-boson mean field method in Ref. [11]. As demonstrated, the $d=1$ diagrams at $n=1$ are occupied by at least nine distinct orderings. Among them: charge-density-waves and superconducting states, site- and bond-located antiferromagnetic phases with their mixed states as well. With increasing lattice dimensionality, the bond-type orderings gradually disappear at $d=\infty$.

The $d=1$ PK model $T=0$ phase diagram obtained within the HFA at $n=1$ [11] is consistent with the respective diagram derived within exact Lanczos diagonalizations [27, $39]$, density matrix renormalization group method [25, 26] and the continuum limit field theory calculations [9, 39]. For $J>0$, these approaches yield the continuous 2ndorder transition to s-wave pairing phase at $J=0^{+}$without any additional transition within the entire range of $J>0$ interaction. This behaviour, at least for alternating lattices, is band-filling independent and it is retained for higher dimensions (including the exactly solvable case of $d=\infty$ ) [11].

We have already partially studied the superconducting characteristics of the PK and PKH models for the case of s-wave (Cooper-pair centre-of-mass momentum $\mathbf{q}=0$ ) $[12,13,18,40,41]$ and eta-phase $(\mathbf{q}=Q$ [16-18] superconductivity. Preliminary investigation of the nextnearest neighbour hopping $t_{2}$ influence on both eta- and s-phase superconductivity in the 2-dimensional PK model has been also evaluated [18]. We have examined in that paper, the evolutions of the $J$ vs. $n$ phase diagrams and determined the $T_{c}$ and $T_{K T}$ transition temperatures with $n$ and $J$. We have also derived, for $t_{2} \neq 0$, the Uemura-type plots for the s-phase.

We have analysed, for the PKH model on the nonfrustrated $d=2 \mathrm{SQ}$ lattice, $T=0$ phase diagrams involving homogeneous magnetic, charge-ordered and superconducting phases [23, 42, 43]. Moreover, within the variational approach (where the $U$ term is treated exactly), the superconducting characteristics (for both s-wave and eta-phase) in the zero-bandwidth limit have been analysed [2, 6, 16, 19, 44].

In this work we extend our studies of the PKH model and analyse in details the evolution of the model's properties for arbitrary concentration $(0<n<2)$ for the attractive $J(J$ $>0)$. Taking into account both the magnetic orderings and superconducting s-wave states, we investigate the impact of the on-site $U$ interaction on the phase diagrams both at $T=0$ and $T>0$. The ground state diagrams involving only homogeneous phases are compared with those involving phase separated states. In both types of diagrams we mark a crossover to the Bose-Einstein Condensate (BEC) regime. Moreover, we present here an extensive analysis of the $U$ interaction effect on the electromagnetic as well as thermodynamic characteristics of the s-phase. The evolutions of the properties with $n$ and interaction parameters are determined. We compare the theoretical London penetration depth $\lambda(n)$ plots with experimental measurements reported by Locquet [48] for $\mathrm{La}_{2-x} \mathrm{Sr}_{x} \mathrm{CuO}_{4}$ films.

For the PKH model on the 2-dimensional lattice, within the Kosterlitz-Thouless (KT) scenario, we investigate the phase fluctuation impact on the s-phase. The $d=2$ KT transition temperature $\left(T_{K T}\right)$ is calculated from the superfluid stiffness $\rho_{s}$ evolution with temperature $T$, which is compared to the KT relation between $\rho_{s}$ and $T_{K T}$. The KT scenario has already given reliable results for the models with attractive intersite density-density interactions [50, 52, 53] and the $d=2 \mathrm{AH}$ model [31, 33]. In the former case a correct behaviour of $T_{K T}$ vs. $-U-/ B$ in agreement with the available Quantum Monte Carlo (QMC) data was found [33]. The $T_{K T}$ is compared with the critical temperature $T_{c}$ obtained within BCS-HFA. The gap to critical temperature ratio is determined and the Uemura-type "universality plots" [55, 57]: $T_{K T}$ vs. $\rho_{S}(0)$ derived within the KT theory are examined. The theoretical Uemura-type plots are compared with the experimental data corresponding to Chevrel phases, doped $\mathrm{BaBiO}_{3}$ (alternating cubic lattices) and cuprates (SQ lattice) [58, 59].

The superfluid properties of the PKH model were studied within a linear response theory [1, 30, 60, 62, 64], where the electromagnetic kernel was treated within the HFARandom Phase Approximation (HFA-RPA) scheme. The HFA-RPA approach is known to be reliable in the case of Hubbard model and its various extensions in the area of the ordered states studied at $T=0$, within the whole range of interaction interpolating between the weak and strong coupling limit $[1,11,30]$. Furthermore, for the electronic models with exclusively intersite interactions in the $d=\infty$ limit, the HFA leads to exact solutions for any For $d<\infty$ at $T>0$, the HFA is much less reliable especially in the strong coupling limit and in low-dimensional systems as it neglects short-range correlations and phase fluctuation effects. 
Hence the HFA neglects phase fluctuation effects and short-range correlations, it is much less reliable in $d<\infty$ at $T>0$, especially for low-dimensional systems in the strong coupling limit.

In a separate work we will study the case of the PKH model with $J<0$, which can stabilize eta-pairing.

Organization of the paper is as follows. In Section 2 there are the electromagnetic kernels and basic equations calculated to determine the basic properties of the studied system in the normal and s-wave superconducting phases. In Sections 3, 4, and 5 we present the numerical analysis of the system. The phase diagrams at $T=0$ involving s-wave superconducting phase as well as nonordered, antiferromagnetic and ferromagnetic states are given in Section 3.1, and the $T>0$ phase diagrams are analysed in Section 3.2. The ground state s-wave phase properties as a function of concentration and coupling strength for SQ and SC structures are shown in Section 4. In Section 5 we devote to superconducting properties analysis at the finite temperatures for the SQ lattice. Going beyond the HFA we calculate the KT critical temperatures $T_{K T}$ and compare them with $T_{c}$ obtained from BCS-HFA. In Section 5. we also demonstrate the Uemura-type "universality plots". Finally, Section 6 contains concluding remarks. In addition, Appendix A is devoted to free energies and self-consistent equations for the phases competing with s-wave paring.

\section{General Formulation}

Taking into account the electrons coupling to magnetic field via the vector potential, the PKH model Hamiltonian takes the following form [9, 11-13, 29, 35]:

$$
\begin{aligned}
H= & -\sum_{i j \sigma} t_{i j}\left(e^{i \Phi_{i j}} c_{i \sigma}^{+} c_{j \sigma}+\text { h.c. }\right)+ \\
& -\sum_{i \sigma} \mu n_{i \sigma}+U \sum_{i} n_{i \uparrow} n_{i \downarrow}+ \\
& -\frac{1}{2} J \sum_{<i j>}\left(e^{2 i \Phi_{i j}} c_{i \uparrow}^{+} c_{i \downarrow}^{+} c_{j \downarrow} c_{j \uparrow}+\text { h.c. }\right),
\end{aligned}
$$

where the Peierls factors incorporate electrons coupling to magnetic field via its vector potential $\mathbf{A}(\mathbf{r})$ (diamagnetic effect):

$\Phi_{i j}=-\frac{e}{\hbar c} \int_{R_{i}}^{R_{j}} d \mathbf{r A}(\mathbf{r}), f$

$e$ is the electron charge, and $<i j>$ limits the sum to nearest neighbours (nn). Other denotations are given after (1).

Following our earlier works on this matter we assume that the effective (phenomenological) model parameters $t$, $U$ and $J$ contain all possible renormalizations and contributions including those coming from the strong electronphonon or from electrons and other electronic subsystems in chemical complexes or solid couplings [1] (e.g. coupling of electrons with intermolecular vibrations via modulation of the hopping integral [65] or the on-site hybridization in a generalized periodic Anderson model [67, 68]). In general, their values and signs could be arbitrary. Particularly interesting is the role of $J$ interaction in a multiorbital model, because of its presence in the iron pnictides [70]. In the strong interaction limit, the $J$ term may also be included in the effective models for Fermi gas on an optical lattice [69].

The self-consistent equations for electron thermal averages and free energy of the PKH model (1) can be determined (within the HFA approximation) by the standard equation of motion or Greens functions approaches [11].

The s-wave phase superconducting order parameter

$x_{s}=\frac{1}{N} \sum_{i}\left\langle c_{i \downarrow} c_{\iota \uparrow}\right\rangle=\frac{1}{N} \sum_{k}\left\langle c_{-k \downarrow} c_{k \uparrow}\right\rangle$,

the Fock term

$p=-1 / 4 N \sum_{k \sigma} \gamma_{k}\left\langle c_{k \sigma}^{+} c_{k \sigma}\right\rangle$,

where $\gamma_{k}=2 \sum_{\alpha} \cos k_{\alpha}, \alpha=x, y, \ldots$, and $\mu$ are determined by the set of equations:

$\frac{\partial F_{S}}{\partial x_{s}}=0, \quad \frac{\partial F_{S}}{\partial p}=0, \quad \frac{\partial F_{S}}{\partial \mu}=0$.

Free energy of the superconducting ( $\mathrm{S}$ ) phase is derived as:

$$
\begin{aligned}
\frac{F^{S}}{N}= & \bar{\mu}(n-1)+\frac{4}{z} J p^{2}+\frac{U n^{2}}{4} \\
& +\left(J_{0}-U\right) x_{s}^{2}-\frac{2}{\beta N} \sum_{\mathbf{k}} \ln 2 \cosh \frac{\beta E_{\mathbf{k}}}{2} .
\end{aligned}
$$

The explicit form of the self-consistent equations determining $x_{s}, p$ and $\mu$ is the following:

$$
\begin{aligned}
& x_{s}=\frac{J_{0}-U}{N} \sum_{k} \frac{x_{s}}{2 E_{k}} \tanh \left(\frac{\beta E_{k}}{2}\right), \\
& p=\frac{-1}{N} \sum_{k} \gamma_{k} \frac{1}{2 E_{k}} \tanh \left(\frac{\beta E_{k}}{2}\right), \\
& n-1=-\frac{2}{N} \sum_{k} \frac{-}{2 E_{k}} \tanh \left(\frac{\beta E_{k}}{2}\right),
\end{aligned}
$$

where $E_{k}=\sqrt{\frac{-2}{k}+\left(-U+J_{0}\right)^{2} x_{s}^{2}}$ is a quasiparticle energy, ${ }_{k}={ }_{k}-\bar{\mu}$, chemical potential: $\bar{\mu}=\mu-\frac{1}{2} U n, N$-number of sites in the system, $n=\frac{N_{e}}{N}(0<n<2)$ denotes number of electrons per site, $J_{0}=z J,{ }_{k}=-\tilde{t} \gamma_{k}, \tilde{t}=t+2 \mathrm{pJ} / z, z$ is the number of nearest neighbours $(z=6$ and $z=4$ for SC and SQ structures respectively), $\beta=1 / k_{B} T$. 
Free energy of the normal $(\mathrm{N})$ phase has the following form:

$$
\begin{aligned}
\frac{F^{N}}{N}= & \bar{\mu}_{N}(n-1)+\frac{4}{z} J p_{N}^{2}+\frac{U}{4} n^{2}+ \\
& -\frac{2}{\beta N} \sum_{\mathbf{k}} \ln 2 \cosh \frac{\beta_{\mathbf{k}}}{2},
\end{aligned}
$$

where $\bar{\mu}_{N}$ and $p_{N}$ are determined as follows:

$n=\frac{1}{N} \sum_{\mathbf{k}}\left(1-\tanh \frac{\beta_{\mathbf{k}}}{2}\right)$,

and

$p_{N}=\frac{1}{N} \sum_{k} \gamma_{k} \frac{1}{2} \tanh \left(\frac{\beta_{\mathbf{k}}}{2}\right)$.

The equations of the other phases considered in this work are given in Appendix A. However, it should be underlined that all results presented in this work are obtained neglecting effects of the Fock term in all phases, i.e., assuming that $p=0$ in each phase.

Based on the linear response theory calculations in the weak vector potential $\mathbf{A}(\mathbf{r})$ case, the expected value of the total current operator Fourier transform in the direction $\alpha(\alpha$ $=x, y, z)[12,13,60,71]$ takes the given form:

$$
\begin{aligned}
J_{\alpha}(\mathbf{q}, \omega)= & N \frac{c}{4 \pi} \sum_{\beta}\left[\delta_{\alpha \beta} K_{\alpha}^{\text {dia }}\right. \\
& \left.+K_{\alpha \beta}^{\text {para }}(\mathbf{q}, \omega)\right] A_{\beta}(\mathbf{q}, \omega) .
\end{aligned}
$$

The total reaction kernel $K_{\alpha \beta}(\mathbf{q}, \omega)$ has been split up into the paramagnetic and diamagnetic parts, which were subsequently evaluated within the RPA-HFA scheme.

In particular, we get the diamagnetic part as follows:

$$
\begin{aligned}
K_{\alpha}^{d i a}= & \frac{8 \pi e^{2}|t|}{\hbar^{2} c^{2} a} \frac{1}{N} \sum_{k}\left[1-\frac{k}{E_{k}} \tanh \left(\frac{\beta E_{k}}{2}\right)\right] \cos \left(k_{\alpha}\right) \\
& -\frac{32 \pi e^{2}}{\hbar^{2} c^{2} a} \frac{J_{0}}{z} x_{s}^{2}
\end{aligned}
$$

where $x_{s}, p$ and $\mu$ are given by (9)-(11).

The expression for the paramagnetic part of the kernel is calculated to be

$$
\begin{aligned}
K_{\alpha \beta}^{p a r a}(\mathbf{q}, \omega)= & -\frac{1}{N} \frac{4 \pi e^{2} t^{2}}{2 \hbar^{2} c^{2}} \sum_{\mathbf{k}} 4 \sin \mathbf{k}_{\alpha} \sin \mathbf{k}_{\beta} \\
& \times\left[\left(\frac{1}{\omega-\left(E_{k-q}+E_{k}\right)+\mathrm{i} \varepsilon}-\frac{1}{\omega+E_{k}+E_{k-q}+\mathrm{i} \varepsilon}\right)\right. \\
& \times\left(1-\frac{\overline{k k-q}+\left(-U+J_{0}\right)^{2} x_{s}^{2}}{E_{k} E_{k-q}}\right) \\
& \times\left[1-n_{F}\left(E_{k}\right)-n_{F}\left(E_{k-q}\right)\right] \\
& +\left(\frac{1}{\omega-\left(E_{k-q}-E_{k}\right)+\mathrm{i} \varepsilon}-\frac{1}{\omega+E_{k-q}-E_{k}+\mathrm{i} \varepsilon}\right) \\
& \left.\times\left(1+\frac{k k-q+\left(-U+J_{0}\right)^{2} x_{s}^{2}}{E_{k} E_{k-q}}\right)\left[n_{F}\left(E_{k}\right)-n_{F}\left(E_{k-q}\right)\right]\right]
\end{aligned}
$$

where $n_{F}\left(E_{\mathbf{k}}\right)$ is the Fermi-Dirac distribution function.
Within London limit the magnetic penetration depth $\lambda$ is defined from the transverse part of the total kernel which in the static limit reads as follows:

$\lambda(T)=\lim _{q_{y} \rightarrow 0}\left[-K_{x}^{\text {dia }}-K_{x x}^{\text {para }}\left(q_{x}=0, q_{y}, q_{z}=0, \omega=0\right)\right]^{-1 / 2}$,

where $K_{x}^{d i a}$ is given by (15) and for the transverse part of the paramagnetic kernel in the static limit for $\mathbf{q} \rightarrow 0$ we obtain:

$K_{x x}^{\text {para }}(\omega=0)=\frac{8 \pi e^{2} t^{2}}{\hbar^{2} c^{2}} \frac{1}{N} \sum_{\vec{k}} \frac{\sin ^{2} k_{x}}{k_{B} T \cosh ^{2}\left(\frac{\beta E_{k}}{2}\right)}$.

At $T=0 \mathrm{~K}$ the paramagnetic part is significant when calculating $\lambda$ for non-local superconductors (Pippard superconductors), common among systems with low $T_{c}$. The materials with short coherence length, including superconductors with high $T_{c}$, represent the so-called London limit. For $\mathbf{q} \rightarrow 0$ and at $T=0$ the magnetic penetration depth in the London limit is expressed with the diamagnetic component of the total kernel:

$\lambda=\frac{1}{\sqrt{-K^{d i a}}}$.

We restrict our investigation to analyzing the system in the London limit in which we can apply the local approximation. Derived for this case $\lambda$ is qualitatively well represented in the weak and strong interactions, moreover, for strong couplings, the results obtained in HFA coincide with those obtained from the perturbation theory.

Having the difference between the normal and superconducting phase free energys and the value of penetration depth we can derive formuls for the thermodynamic critical field $H_{c}$ and the Ginzburg-Landau correlation length $\xi_{G L}$ :

$$
\begin{aligned}
\frac{H_{c}^{2}(T)}{8 \pi} & =\frac{F^{N}(T)-F^{S}(T)}{N a^{3}}, \\
\xi_{G L} & =\frac{\Phi_{0}}{2 \pi \sqrt{2} \lambda H_{c}},
\end{aligned}
$$

where $a$ is the lattice constant, $\Phi_{0}=\frac{h c}{2 e}$, and we get the estimations for the critical fields:

$H_{c 1} \simeq \frac{\ln \kappa}{\kappa} H_{c} ;$

$H_{c 2}=\Phi_{0} /\left(2 \pi \xi_{G L}^{2}\right)$,

where $\kappa$ is the Ginzburg ratio: $\kappa=\lambda / \xi_{G L}$.

At $T=0, \frac{F(0)}{N}=E_{0}=\frac{1}{N}\langle H\rangle_{(T=0)}$. The HFA expressions determining normal and superconducting ground state energies, $E_{0}^{N}$ and $E_{0}^{S}$ are:

$E_{0}^{N}=\frac{F^{N}(0)}{N}=E_{N}^{K}(0)+\frac{4}{z} J p_{N}^{2}+\frac{1}{4} U n^{2}$, 


$$
\begin{aligned}
E_{0}^{S}= & \frac{F^{S}(0)}{N}=E_{S}^{K}(0) \\
& +\frac{4}{z} J p^{2}+\frac{\left(-U+J_{0}\right)^{2} x_{S}^{2}}{U-J_{0}}+\frac{1}{4} U n^{2},
\end{aligned}
$$

where

$$
\begin{aligned}
E_{\mathrm{N}(\mathrm{S})}^{K} & =\frac{1}{N} \sum_{\mathbf{k} \sigma} \mathbf{k}_{\mathbf{k} \sigma}\left\langle c_{\mathbf{k} \sigma}^{+} c_{\mathrm{N}(\mathrm{S})},\right. \\
\left\langle c_{\mathbf{k} \sigma}^{+} c_{\mathbf{k} \sigma}\right\rangle_{S} & =\frac{1}{N} \sum_{\mathbf{k}}\left(1-\frac{\mathbf{k}}{E_{\mathbf{k} \sigma}}\right), \\
\left\langle c_{\mathbf{k} \sigma}^{+} c_{\mathbf{k} \sigma}\right\rangle_{N} & =\frac{2}{N} \sum_{\mathbf{k}} \operatorname{sign}(\mathbf{k}-\bar{\mu}),
\end{aligned}
$$

$E_{\mathrm{S}(\mathrm{N})}^{K}$ is the mean kinetic energy of the superconducting (normal) phase.

From (9)-(11) and the condition $\Delta \rightarrow 0$, where gap amplitude parameter: $\Delta=\left(-U+J_{0}\right) x_{s}$, one can calculate the HFA transition temperature $T_{c}$ which is an estimation of the temperature of pair-formation [12, 33, 52].

Due to the phase fluctuation effects, for finitedimensional lattice $(d<\infty)$, transition to the superconducting phase occurs at temperature lower than $T_{c}$ (HFA). We take into account the phase fluctuation effects by determining another characteristic temperature, the KosterlitzThouless critical temperature $\left(T_{K T}\right)$. For two-dimensional lattices $(d=2)$ the value of $T_{K T}$ can be derived within the KT theory $[12,31,33,72,73]$. In terms of the theory the $T_{K T}$ temperature is defined by vortex pair unbinding transition determined by universal jump of the superfluid stiffness (helicity modulus) $\rho_{s}$ at $T_{K T}$ :

$k_{B} T_{K T}=Q \rho_{s}\left(T_{K T}\right)$,

where $Q \simeq 0.898$ (Monte-Carlo estimates for $d=2$ $\mathrm{XY}$ model $[33,72])$. The superfluid stiffness $\rho_{s}$, which is directly related to the London penetration depth $\lambda$ and calculated within HFA-RPA scheme using (15), (18) and (17) has the following explicit form:

$$
\begin{aligned}
\rho_{s}(T)= & \frac{\hbar^{2} c^{2}}{16 \pi e^{2}} \lambda^{-2}=\frac{-\hbar^{2} c^{2}}{16 \pi e^{2}}\left[K_{x}^{\text {dia }}+K_{x x}^{\text {para }}(\omega=0)\right] \\
= & -\frac{1}{2 N} \sum_{k}|t|\left[1-\frac{\underline{k}}{E_{k}} \tanh \left(\frac{\beta E_{k}}{2}\right)\right] \cos \left(k_{\alpha}\right) \\
& +2 \frac{J_{0}}{z} x_{s}^{2}-\frac{1}{2 N} t^{2} \sum_{k} \frac{\sin ^{2} k_{x}}{k_{B} T \cosh ^{2}\left(\frac{\beta E_{k}}{2}\right)} .
\end{aligned}
$$

Thus, $T_{K T}$ is determined by (9)-(11) and (26) with $\rho_{s}$ given by (27). Since the formula (27) does not take into account the renormalization coming from the topological excitations (vortex-antivortex pairs) [31,33], calculated this way $T_{K T}$ is only an upper bound estimation of the actual KT transition temperature.
Using our approach one is able to analyse the crossover between BCS-like (extended Cooper pairs ("BCS")) and local Cooper pairs superconductivity (composite bosons, Bose-Einstein Condensate (BEC)). The crossover takes place when increasing pairing interaction $(J$ or $-U-$ $(U<0))$ one increases coupling from a weak to strong regime. At the ground state, one can approximate boundary between both regimes from the Leggett criterion [30, 74], which requires the chemical potential of the superconducting phase to be located at the bottom of the electronic band, i.e.:

$\bar{\mu}_{s}=-B / 2$,

where $B$ is the bandwidth of $k_{k}(B=2 z t)$ and $\mu_{s}$ is determined from the self-consistent (9)-(11) solved at $T=0$.

\section{Phase Diagrams of the Model at $T \geq 0$}

We performed extensive analytical and numerical analysis of the electromagnetic and thermodynamic properties of the s-wave phase of the PKH model (3) for $d$-dimensional hypercubic lattices.

In this Section we discuss the phase diagrams for arbitrary $n$ and interaction parameters, determined within the HFA in the ground state (Sec. A) and $T>0$ (Sec. B), whereas the superfluid characteristics of $s-$ wave phase are analysed in Sections 4 and 5.

For $d=2$ we used the square lattice (SQ) density of states expressed with:

$D()_{2 D}=\frac{1}{2 t \pi^{2}} K\left(1-\left(\frac{-}{4 t}\right)^{2}\right)$,

if $-/ 4 t-<1$ and zero otherwise, where $K$ is the complete elliptic integral of the first kind. For $d=3$ we use the analytical approximation to $D()$ as calculated numerically by Jelitto [75].

\subsection{Ground State Phase Diagrams}

Below we analyse phase diagrams at $T=0$ for the SQ lattice involving the homogeneous: nonordered $(\mathrm{N})$, swave pairing (S), ferromagnetic (F) and antiferromagnetic (AF) states and compare them with the phase diagram involving also phase-separated states. We only analyse the simple magnetic phases but consideration of more complex magnetic orderings would not modify significantly the results. In general, in the ground state, the $\mathrm{S}$ states are always stable with respect to the $\mathrm{N}$-phase for $J>U / z$ for arbitrary values of single electron hopping, $U, J$ and $n$ $(0<n<2)$. In the figures we mark the crossover to the Bose-Einstein Condensate (BEC) which at $T=0$ can be located from Leggett's criterion [30, 74] (28). For selected 
interaction values we also plot the evolution of the order parameters of the respective phases as a function of $n$.

Figures 1 and 2 present the $T=0$ phase diagrams $U$ versus $n($ for $J / 4 t=1)$ and $J$ versus $n$ (for $U / 4 t=2$ and $U / 4 t=3$ ) respectively. The transition lines to $\mathrm{N}$ phase are of the $2 \mathrm{nd}-$ order, while those between the ordered states are of the 1st-order. As a result of competition between repulsive $U$ and $J$, the s-wave pairing state is limited on the diagram, Fig. 1, for $U<U_{c}$ (with $U>0$ ), whereas the $\mathrm{F}$ and $\mathrm{AF}$ phases are stable for $U$ above $U_{c}\left(U>U_{c}\right)$. The $\mathrm{F}$ and AF phases occur in the diagram in the limited ranges of concentration $n$ : for low and intermediate values of $n$ and close to half-filling $(n=1)$, respectively. For $U<U_{c}(n=1)$ states S may occur within entire range of $n$. Because of the strong interactions the nonordered phase is not present on the phase diagram. For given concentration $n$, with increasing on-site $U$ interaction, system exhibits several sequences of transitions: (i) a single 1st-order transition $\mathrm{S}$ $\rightarrow \mathrm{F}$, (ii) a sequence of 1st-order transitions $\mathrm{S} \rightarrow \mathrm{AF} \rightarrow \mathrm{F}$ (with increasing $U, \mathrm{AF} \rightarrow \mathrm{F}$ transition line asymptotically approaches half-filling) and (iii) at $n=1$, a single 1st-order transition $\mathrm{S} \rightarrow \mathrm{AF}$.

The $J$ vs. $n$ phase diagrams, for fixed $U$ at $T=0$ is shown in Fig. 2. Analogously to the previous diagram (Fig. 1), the S-phase may occur in the diagram above a critical $J_{c}(U, n)$. Increasing $U$ reduces concentration ranges occupied by the $\mathrm{AF}$ and $\mathrm{N}$ phases and expands the range of $\mathrm{F}$ state stability in the diagram (comp. Fig. 2a and b). In both diagrams, with increasing $J$, the system can exhibit several different types of behaviour, depending on $n$ : (i) a single 1st-order transition $\mathrm{N} \rightarrow \mathrm{S}$, (ii) a single 1st-order transition $\mathrm{F} \rightarrow \mathrm{S}$ and (iii) a single 1st-order transition $\mathrm{AF} \rightarrow \mathrm{S}$.

Figure $3 \mathrm{~b}$ presents the phase diagram $U$ versus $n$ (for $J / 4 t=1.0$ ) involving the phase-separated states (PS). This diagram is a preliminary result, a part of a broader analysis of phase diagrams involving PS states, which is soon to be published elsewhere.. In the figures presented here, for the sake of clarity, the $\mu$-dependent plot (Fig. 3a) is given, which helps to determine boundaries between the PS states plotted in Fig. 3b. All homogeneous ordered states are separated by PS states (Fig. 3b). The transitions between homogeneous and the PS states are symbolically named "third order" transitions [6]. In the system that undergoes this type of transition, one of the domains in the PS phase gradually vanishes.

In analogy to earlier presented diagrams involving homogeneous states only (cf. Fig. 1), also in this case (Fig. 3b) the states involving s-wave pairing are stable only below certain critical values $U_{c}$. For $U>U_{c}(n$ $=1$ ) superconducting $\mathrm{S}$-states can be stable only within a limited range of $n(n<1)$ and appear in the diagram as a homogeneous or phase-separated states involving $\mathrm{AF}$ and $\mathrm{F}$ orderings. At half-filling, for $U<U_{c}$ the S-phase may occur within the whole range of concentrations. The homogeneous AF phase occurs only for $n=1$ when $U>$ $U_{c}$. Away from $n=1$, within a limited range of $U$, the $\mathrm{AF}$ phase may be stable in the diagram exclusively within the $\mathrm{PS}(\mathrm{S} / \mathrm{AF})$ and $\mathrm{PS}(\mathrm{AF} / \mathrm{F})$ states close to $n=1$.

As the electron density increases towards half-filling, depending on $U$, the system exhibits a few distinct transition sequences: (i) a single 1st-order transition $\mathrm{F} \rightarrow \mathrm{PS}(\mathrm{AF} / \mathrm{F})$ $\rightarrow \mathrm{AF}$, (ii) a sequence of four 1st-order transitions: $\mathrm{S} \rightarrow$ $\mathrm{PS}(\mathrm{S} / \mathrm{F}) \rightarrow \mathrm{F} \rightarrow \mathrm{PS}(\mathrm{AF} / \mathrm{F}) \rightarrow \mathrm{AF}$ and (iii) a sequence of two 1st-order transitions: $\mathrm{S} \rightarrow \mathrm{PS}(\mathrm{S} / \mathrm{AF}) \rightarrow$ AF.

In the plots (Figs. 1, 2 and 3) the crossover to BEC regime is denoted with dashed line. An obvious effect of the repulsive (attractive) $U$ is the reduction (expansion) of the composite bosons (BEC) regime. As we see from the plots, with rising concentration $n$, the boundaries are moved towards greater values of interaction $J$ for any $U$.
Fig. 1 The $U$ vs. $n$ phase diagram for fixed $J / 4 t=1$ at $T$ $=0$. Denotations: $s-$ wave superconducting state $(\mathrm{S})$, ferromagnetism $(\mathrm{F})$ and antiferromagnetism (AF). Dashed line denotes crossover to $\mathrm{BEC}$ regime. The phase transtion lines are of the 1st-order. Diagram is plotted for SQ lattice

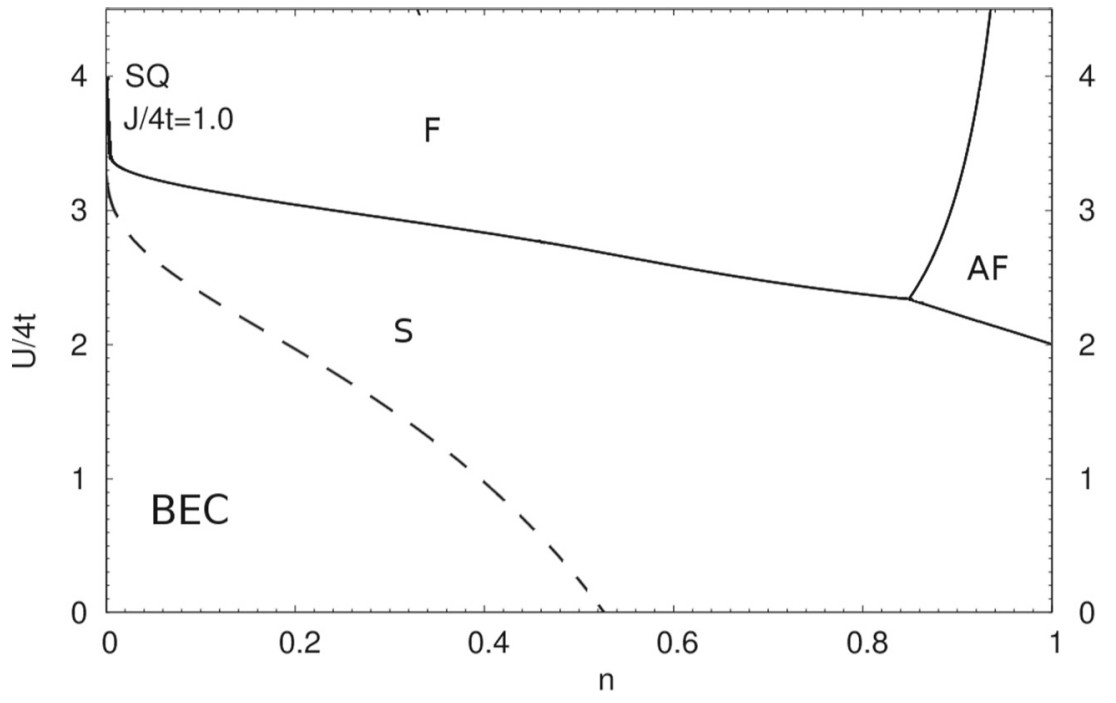



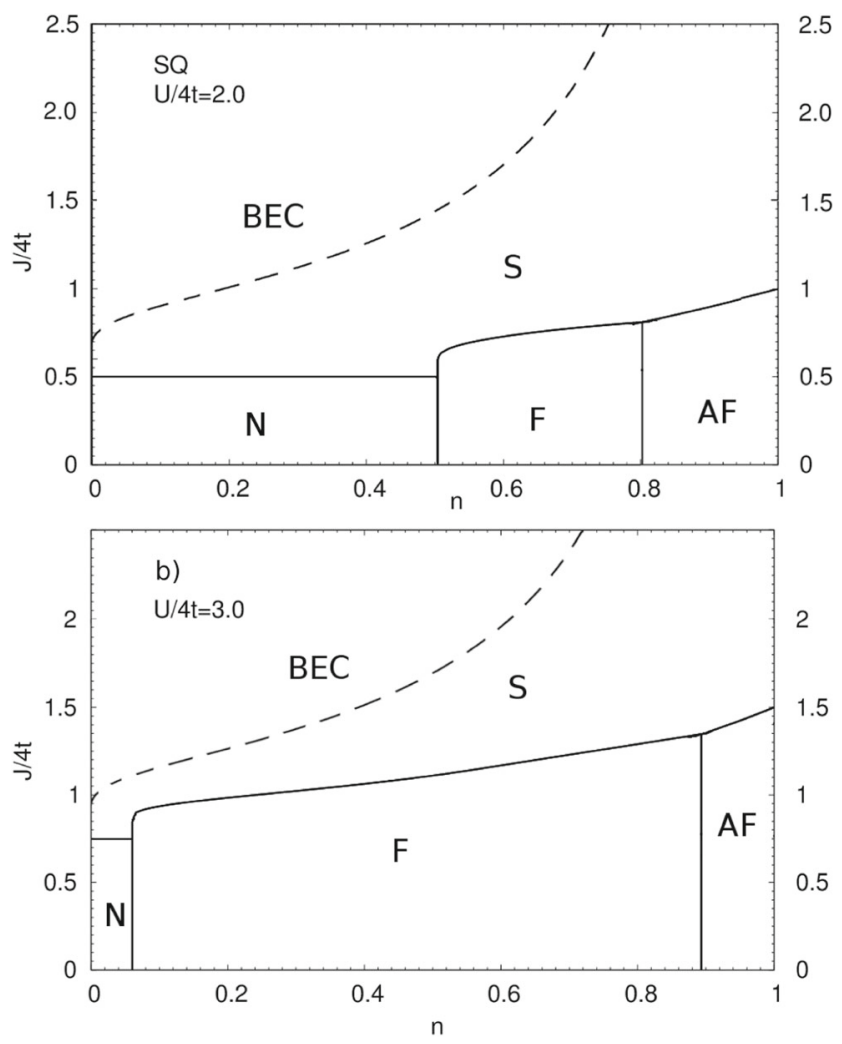

Fig. 2 The $J$ and $n$ phase diagrams plotted for $U / 4 t=2$ and $U / 4 t=3$ at $T=0$. All the transitions involving exclusively ordered states are of the 1st-order and those to nonordered $(\mathrm{N})$ phase are of the 2 nd-order. Diagrams are plotted for SQ lattice. Other denotations as in Fig. 1

Thus, within a definite range of $U$ an $J$, the crossover from the weakly to the strongly coupled electron pairs can be achieved by lowering the electron density.

\subsection{Finite Temperature Phase Diagrams}

In this subsection we present a two examples of the finite temperature phase diagrams, as evolution with concentration (Fig. 4) and evolution with $U$ (Fig. 5) for the repulsive on-site interaction $U>0$.

For interaction parameters $U / 4 t=3.0$ and $J / 4 t=1.0$ (Fig. 4) with increasing temperature the system can exhibit the following phase transitions: (i) a single 2nd-order transition $\mathrm{S} \rightarrow \mathrm{N}$ (ii) a sequence of three transitions: 2 ndorder $\mathrm{S} \rightarrow \mathrm{N}$ and reentrant 2nd-order $\mathrm{N} \rightarrow \mathrm{F} \rightarrow \mathrm{N}$, (iii) a sequence of five transitions: 2 nd-order $\mathrm{S} \rightarrow \mathrm{N}$, reentrant 2nd-order $\mathrm{N} \rightarrow \mathrm{F} \rightarrow \mathrm{N}$, reentrant 2nd-order $\mathrm{N} \rightarrow \mathrm{AF}$ $\rightarrow \mathrm{N}$, (iv) a sequence of 1 st-order transition $\mathrm{F} \rightarrow \mathrm{AF}$ and 2nd-order AF $\rightarrow \mathrm{N}$ and (v) a single 2nd-order transition $\mathrm{AF} \rightarrow \mathrm{N}$.

The diagram $T$ versus $U$ for $J / 4 t=1.0$ and $n=0.9$ is given in Fig. 5. As one can notice from the plot, with increasing $T$ the system can undergo either (i) a single 2nd-order
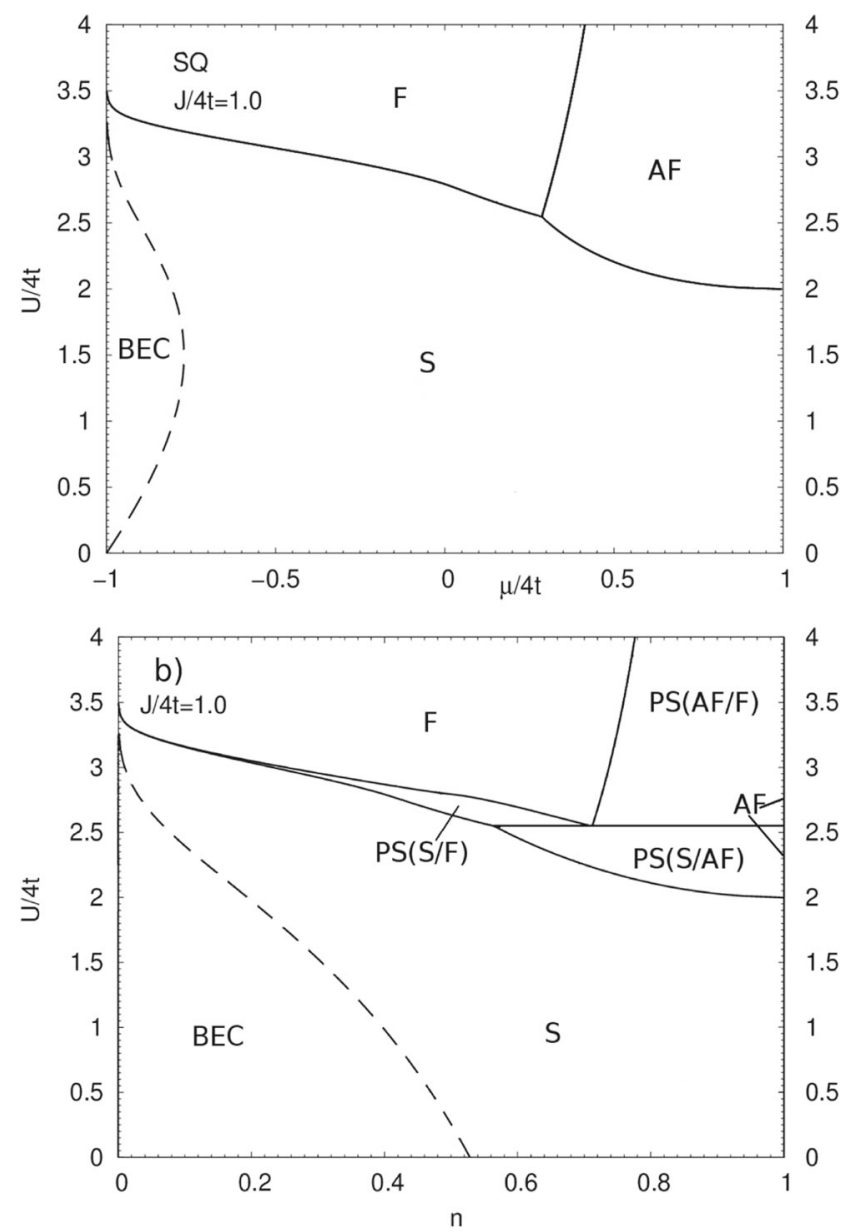

Fig. 3 The $T=0$ phase diagrams as a function of repulsive $U$ vs. a chemical potential $\mu, \mathbf{b}$ carriers density $n$ for fixed $J / 4 t$ $=1$. Denotations: phase separated states (PS), nonordered phase $(\mathrm{N})$, s-pairing superconducting state $(\mathrm{S})$, ferromagnetism $(\mathrm{F})$ and antiferromagnetism (AF). Dashed line denotes the crossover to BEC regime. Diagrams are plotted for SQ lattice

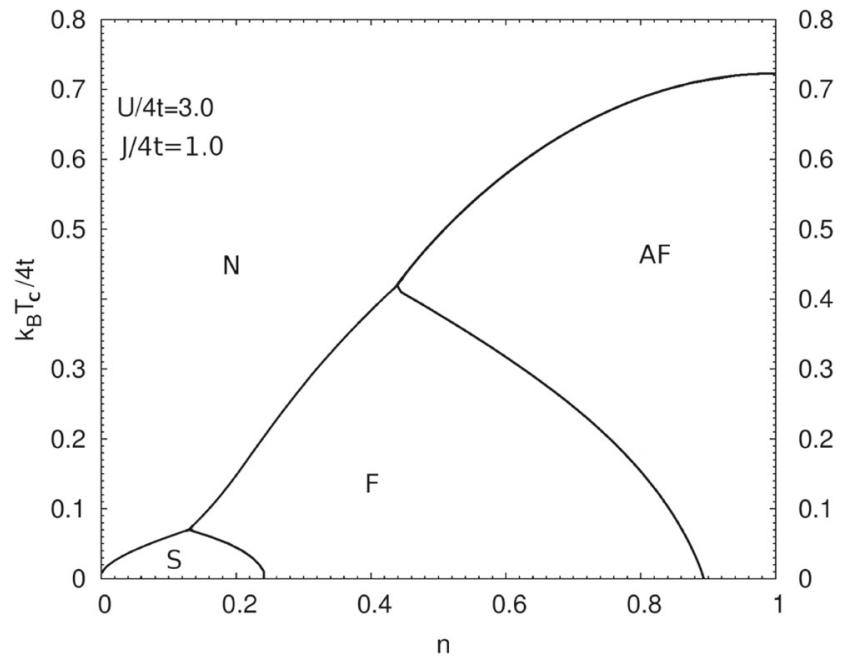

Fig. 4 Finite temperature phase diagram for fixed values of interaction parameters $U / 4 t=3.0$ and $J / 4 t=1.0$. (SQ lattice) 


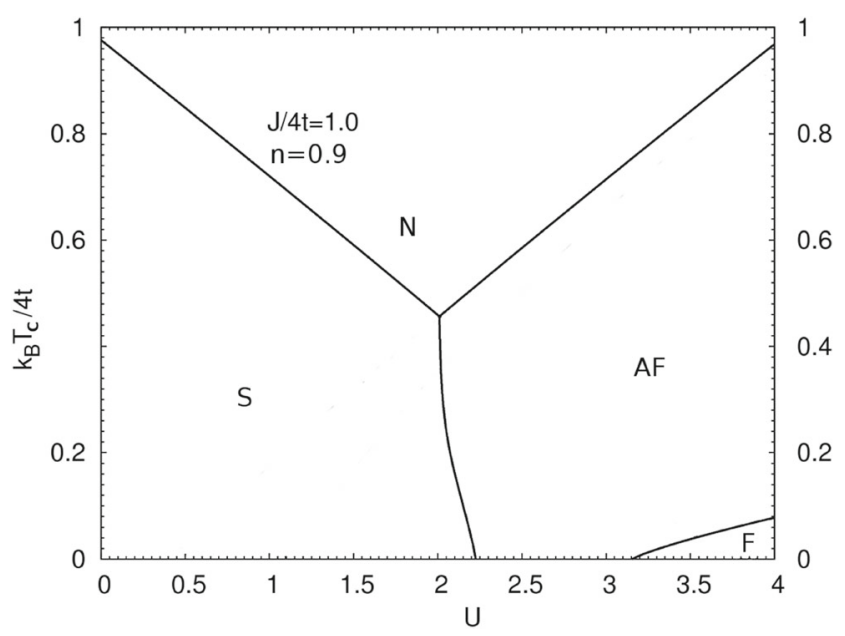

Fig. 5 Finite temperature phase diagram for fixed values of interaction parameters $J / 4 t=1.0$ and $n=0.9$. (SQ lattice)

transition $\mathrm{S} \rightarrow \mathrm{N}$, (ii) a sequence of two transitions: 1storder $\mathrm{S} \rightarrow \mathrm{AF}$ and 2 nd-order $\mathrm{AF} \rightarrow \mathrm{N}$, or (iii) a sequence of two transitions: 1st-order $\mathrm{F} \rightarrow \mathrm{AF}$ and 2 nd-order $\mathrm{AF}$ $\rightarrow \mathrm{N}$.

\section{Superfluid Characteristics of s-wave Phase at $T=0$}

Below we present the evolutions of the ground state superconducting properties of the s-wave phase with model parameters (Figs. 6, 7, 8 and 9). The plots were determined for the parameters' ranges where superconducting S-phase is stable in the phase diagrams analysed earlier in the paper. Due to the electron-hole symmetry of the model all the plots are symmetric with respect to the transformation $n \rightarrow 2-n$. As one can see from the figures (cf. Figs. 1-3), where the superconducting characteristics are plotted as a function of $U / B$ for $J_{0} / B>0$, the $\mathrm{S}$ states can still be stable even for $U$ $>0$ (repulsibe $U$ ) when $J_{0}-U>0$. With increasing $J_{0} / B$ the S-phase boundary shifts towards higher values of $U / B$.

In Fig. 6 we show plots of the inverse square value $\left(1 / \lambda^{2}\right)$ of the London penetration depth and of the order parameter $\Delta$ as a function of $U / B$ for fixed values of $n$ and $J_{0} / B$ at $T$ $=0$ for the SQ and SC lattices. These quantities plotted as a function of $n$ for nonfrustrated 2D SQ lattice and selected values of $J_{0} / B$ and $U / B$ are presented in Fig. 7. As the pairing interactions $J_{0}$ or $-U-$ (for $U<0$ ) increase, the $1 / \lambda^{2}, \Delta$ and other superconducting characteristics evolve smoothly from the limit of weakly interacting single particle carriers (with $\lambda^{-2}$ being proportional to the bandwidth $B$, $\Delta \sim \exp \left(\frac{-2 B}{J_{0}-U}\right)$ if $\left.n \neq 1\right)$ to the limit of tightly bound pairs (with $\Delta \sim J, \Delta \sim|U|$ and $\lambda^{-2} \sim J$ if $U=0$ ). For $J_{0} / B=0$, the PKH model reduces to AH model. In this case

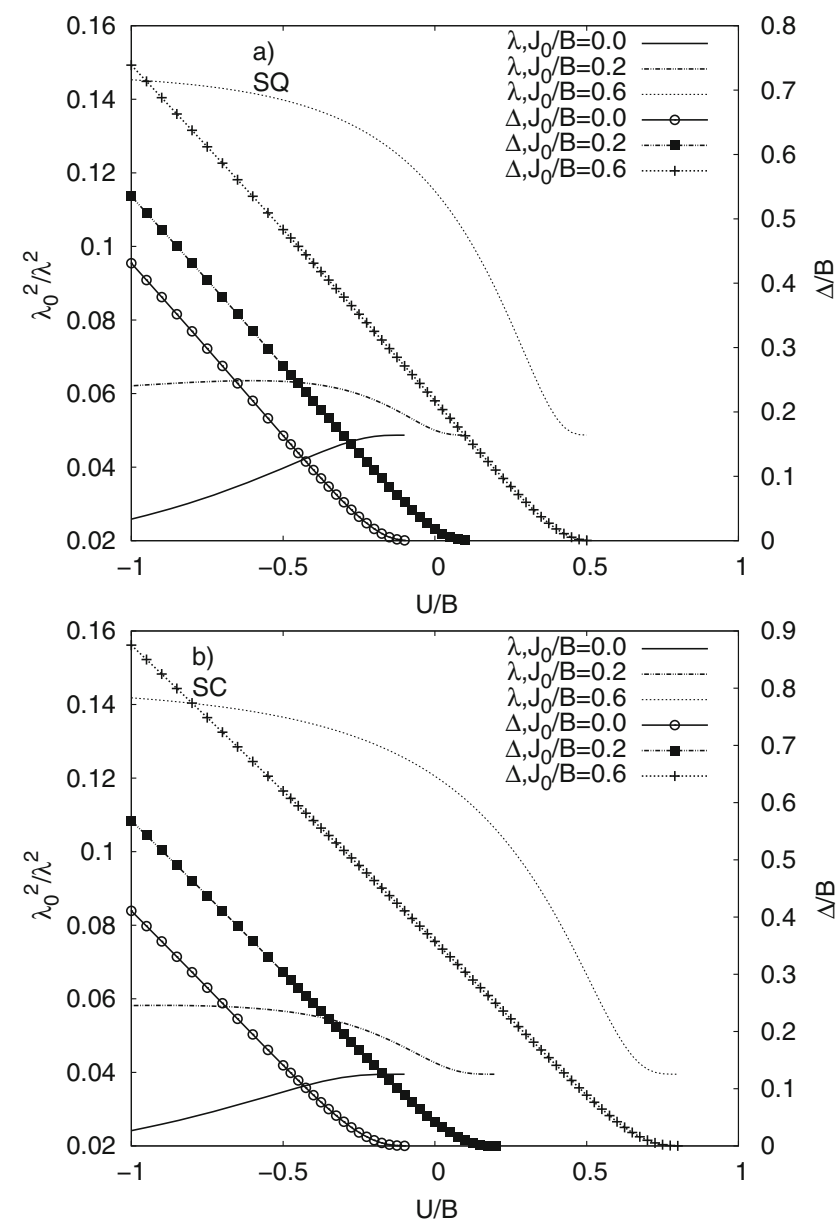

Fig. 6 The inverse square penetration depth $1 / \lambda^{2}$ and the gap parameter $\Delta=\left(J_{0}+U\right) x_{S}$ at $T=0$ as a function of $U / B$ for $n=0.75$ and fixed values of $\mathbf{a} J_{0} / B=0.0 ; 0.2 ; 0.4$ plotted for SQ lattice and $\mathbf{b}$ $J_{0} / B=0.0 ; 0.2 ; 0.6$ plotted for SC lattice. $\lambda_{0}=\frac{\hbar c}{e} \sqrt{\frac{a^{d-2}}{4 \pi \mathrm{B}}}, B=2 z t$ is the bandwidth, $a$ is the lattice constant

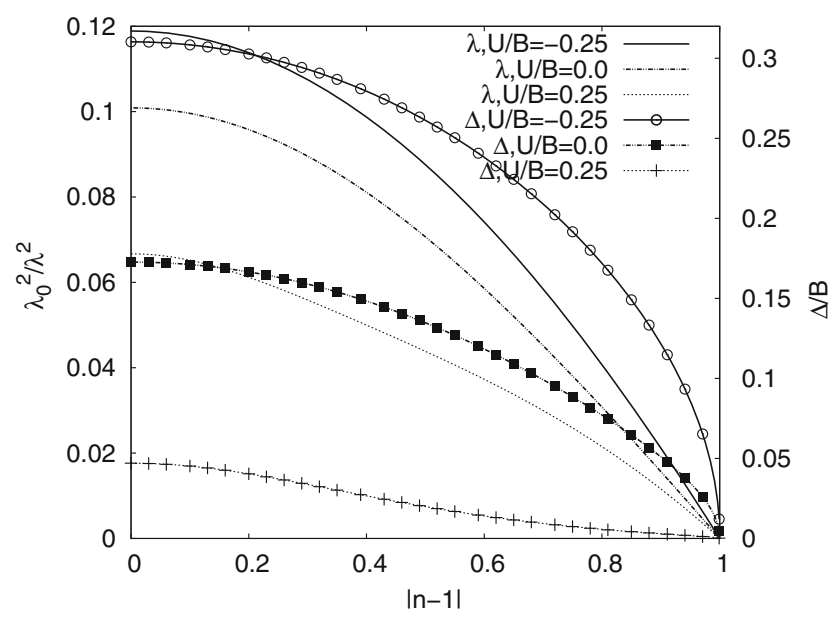

Fig. 7 Concentration dependence of $1 / \lambda^{2}$ and the gap parameter $\Delta$ at $T=0$ for $J_{0} / B=0.5$ and a few values of $U / B: U / B=-0.25 ; 0.0 ; 0.25$ (SQ lattice) 

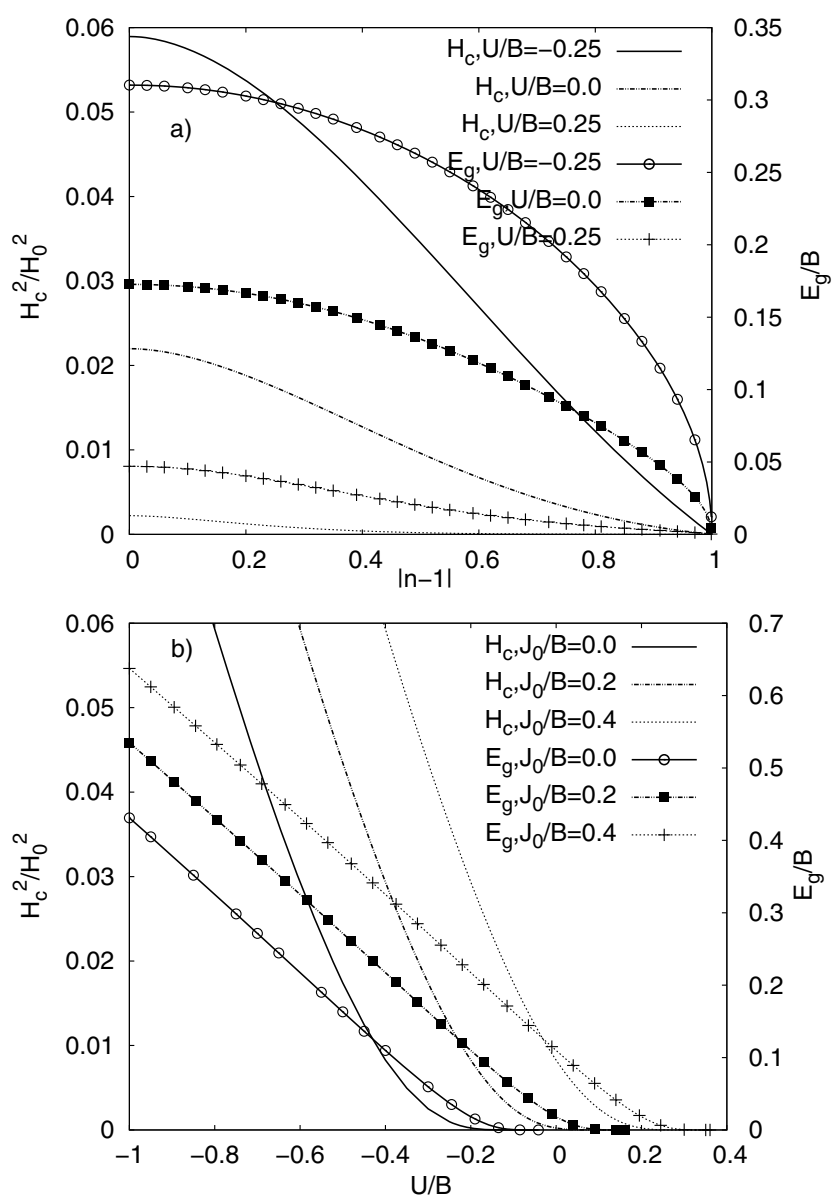

Fig. 8 Concentration $\mathbf{a}$ and $U \mathbf{b}$ dependencies of the reduced square value of thermodynamic critical field $H_{c}^{2} / H_{0}^{2}\left(H_{0}^{2}=4 \pi \mathrm{B} / a^{d}, B\right.$ $=2 z t=8 t)$ and the energy gap $E_{g}(0)=\min E_{k}-\max \left(-E_{k}\right)$ at $T=0$ for a) $J_{0} / B=0.5$ and several values of $U / B: U / B=-0.25, U / B=0.0$, $U / B=0.25$ b) $n=0.75$ and $J_{0} / B=0.0, J_{0} / B=0.2, J_{0} / B=0.4$ (SQ lattice)

$\lambda^{-2}$ continuously decreases with increasing $-U-/ B$ for any $-U-$ and $\lambda^{-2} \sim t^{2} /|U|$ for $-U-\mid B>>1$. For fixed $J_{0} / B>0$ following the increase of $-U / B$, the $\lambda^{-2}$ first grows, and subsequently it passes through a round maximum and drops at high $-U / B$ proportionally to $2 t^{2} /-U-+J$ (cf. Fig. 6). With increasing $J_{0} / B$ the maximum value of $\lambda^{-2}$ increases. Notice that the increase in $\lambda^{-2}$ as a function of $J_{0} / B$ for any value of $U / B$ (the increase is linear for large $J$ ) is significantly distinct form the behaviour observed for the AH model $\left(J_{0}=0, U<0\right)$.

The following figures present the energy gap $E_{g}(0)$ and the behaviour of the square value of thermodynamic critical field $H_{c}^{2}$ at $T=0$ for SQ lattice. The quantities are plotted as a function of $n$ for a fixed value of $J_{0} / B$ and several values of $U / B$ (Fig. 8a) as well as a function of $U$ for a fixed $n$ and several values of $J_{0} / B$ (Fig. 8b). Both characteristics vanish at the empty-band limit $(n=0)$, reach a maximum at half-filling and increase linearly with increasing $-U-/ B$
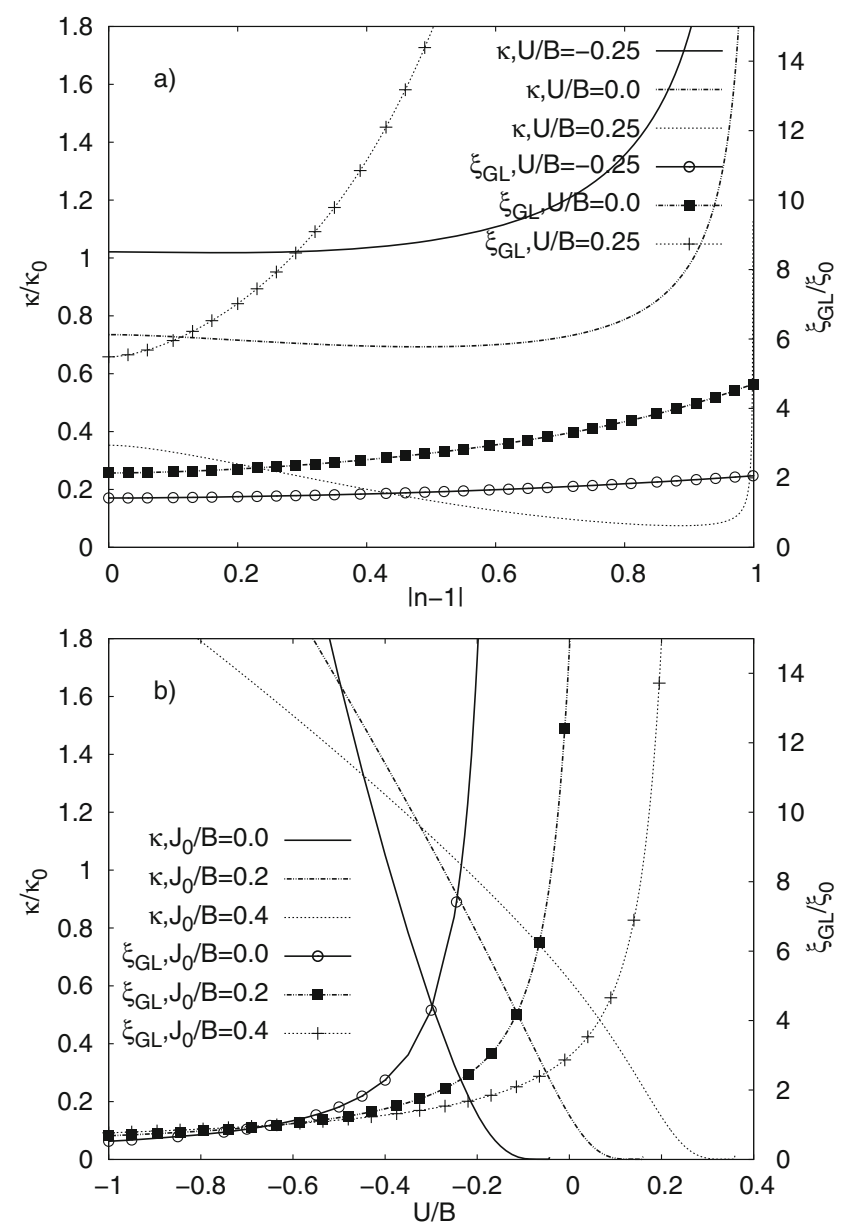

Fig. 9 Concentration a and interaction $U \mathbf{b}$ dependencies of the $\mathrm{G}-\mathrm{L}$ coherence length $\xi_{G L} / \xi_{0}\left(\xi_{0}=\frac{a}{\sqrt{2 z}}\right)$ and the Ginzburg ratio $\kappa / \kappa_{0}($ $\kappa_{0}=\frac{\hbar c}{e} \sqrt{\frac{2}{\pi \mathrm{B} a^{4-d}}}$ ) at $T=0$ plotted for a) $J_{0} / B=0.5$ and $U / B=0.0, U / B$ $=0.25, U / B=-0.25$ and $\mathrm{b}$ ) for $n=0.75$ and $J_{0} / B=0.0, J_{0} / B=0.2$, $J_{0} / B=0.4$. (SQ lattice)

for $U / B>>1$, and in the weak coupling limit they are proportional to $\exp \left(\frac{-2 B}{J_{0}-U}\right)$.

The plots of G-L coherence length $\xi_{G L}$ and the Ginzburg ratio $\kappa$ versus $U / B$ and $n$ calculated for SQ lattice for selected values of respective interaction parameters are presented in Fig. 9. In the limit of weakly interacting single particle carriers the $\xi_{G L} \sim \exp \left[\frac{-2\left(J_{0}-U\right)}{B}\right], \kappa \sim$ $\exp \left(\frac{B}{J_{0}-U}\right)$, and at tightly bound pairs regime, $\xi_{G L} \Rightarrow$ $\frac{a}{\sqrt{2 z}}, \kappa \sim \sqrt{\frac{1}{J}}$ for $U=0$, and $\kappa \sim \sqrt{\frac{1}{2 t^{2} /|U|+J}}$, if $-U-/ B$ $>>1, U<0$ ).

As concerns the concentration dependencies of various quantities (see Figs. 7, 8 and 9) one finds that in the low density limit for arbitrary coupling strengths $\lambda^{-2} \sim n$ (as for the fermions in the continuum), $\Delta \sim \sqrt{n}, H_{c}^{2} \sim n$, $\kappa \sim \frac{1}{\sqrt{n}}$, whereas for $\left(J_{0}-U\right) / B>>1: \Delta \Rightarrow(1 / 2)\left(J_{0}-\right.$ U) $\sqrt{n(2-n)}, \lambda^{-2} \sim n(2-n), H_{c}^{2} \sim n(2-n), \kappa \sim$ 
$\frac{1}{\sqrt{n(2-n)}}$, for any $n$. Beyond these limits the concentration dependencies are not universal. Their behaviour highly depend on the features of density of states. Notice, the resulting from the van Hove singularity in $D($ ) for SQ lattice, substantial enhancement of $\Delta$ and $H_{c}^{2}$ close to $n=1$ (Figs. 7 and 8 ) observed in the range of small and intermediate values of $\left(J_{0}-U\right) / B$. Similar efect is observed for the local maximum of the Ginzburg ratio $\kappa$ at $n=1$ (Fig. 9a). In addition, in Fig. 9a one can see a significant variation of $\xi_{G L}$ as a function of $n$ in the weak and intermediate coupling strength.

\section{Critical Temperatures for s-wave Phase and the Uemura-Type Plots}

Apart form the weak coupling as well as from the case of $d=\infty$, the phase fluctuations exert a strong impact on the superconducting pairing, and the results display a clear energy scales separation for the pair formation ( $\sim k_{B} T_{c}$ ) and phase coherence. The effects of the phase fluctuations are most clearly seen for $d=2$ lattices, whose phase coherence temperature is determined by $k_{B} T_{K T}$ (26), (27).

Between $T_{c}$ and $T_{K T}$ incoherent s-wave phase occurs, where the pseudogap opens up in the quasiparticle energy spectrum and the system exhibits non-Fermi liquid properties. Not at $T_{c}$, but only at $T_{K T}$ the real transition to a superconducting state takes place, where the transition to a phase with bound vortex-antivortex pairs occurs. Certain pairing correlations are observed at all temperatures thus $T_{c}$ does not represent a rigorous boundary line. It is rather a reliable estimate of the pair formation and the pseudogap emergence [12, 30, 36].

For SQ lattice the evolutions of $T_{K T}$ and $T_{c}$ with a change in $n$ for fixed interaction parameters are shown in Fig. 10, whereas Fig. 11 present the plots of transition temperatures as a function of interactions. The $T_{K T}$ temperature can be significantly reduced compared to $T_{c}$, where for $U$ $\leq 0, J_{0}>0$ the highest reduction occurs for low values of carriers density $n$ (cf. eg. Fig. 10 and, for $U=0$, Ref. [12]). Moreover, the difference between $T_{c}$ and $T_{K T}$ strongly increases with the increase of on-site attraction $U$ $<0$ (cf. Fig. 11). Analogously as found for PK [12] and $\mathrm{AH}$ [30] models, also for the PKH model one observes a monotonous increase in $T_{c}$ with increasing interactions, (which is obviously a result of increasing pair binding energy) (cf. Fig. 11 [12]). On the other hand, apart from the weak paring strength regime $T_{K T}$ evolution with interaction parameter is significantly distinct for these models. As we can see in Fig. 11, for the PKH model, similarly as we observe for the AH model, with interaction - $U$ - increase the $T_{K T}$ at first increases exponentially, and subsequently it passes a round maximum and then drops as $t^{2} /-U-$

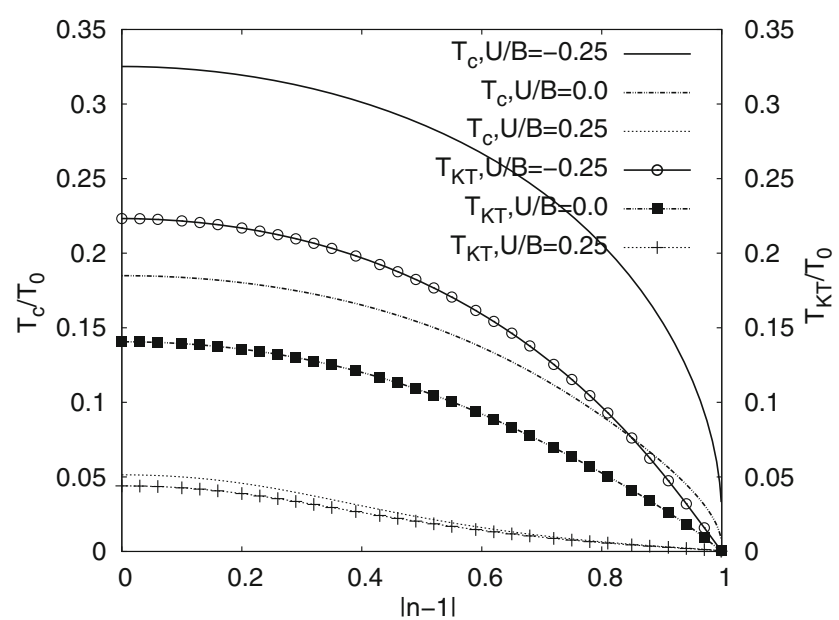

Fig. 10 Concentration dependencies of $T_{K T}$ and $T_{c}$ for SQ lattice plotted for several values of interaction parameters: $J_{0} / B=0.5$ and $U / B$ $=0.0, U / B=0.25, U / B=-0.25 . B=8 t, J_{0}=4 J, T_{0}=\frac{B}{K_{B}}$

at strong $-U$ - Analogous behaviour was found for the thermodynamic critical field $H_{c}(0)$ [12, 30, 33]. On the other hand in the PK model, the model with intersite charge exchange, $T_{K T}$ monotonously increases with $J$, and the growth of $T_{K T}$ is linear at strong values of $J$ (cf. Ref. $[11,12])$. Due to the difference in the paring mechanisms, the dynamics of electron pairs is qualitatively different in both models. [11, 12, 30]. The PKH integrates both of the pairing mechanisms and the interplay between $U$ and $J$ interactions results in the differences in the thermodynamic and electrodynamic properties compared to those in the PK and $\mathrm{AH}$ models.

In contrast to the BCS case, the phase fluctuations enhance the ratio of the ground state gap $\left(E_{g}=\min E_{k}\right.$ $\left.-\max \left(-E_{k}\right)\right)$ to the critical temperature. In Fig. 12a we

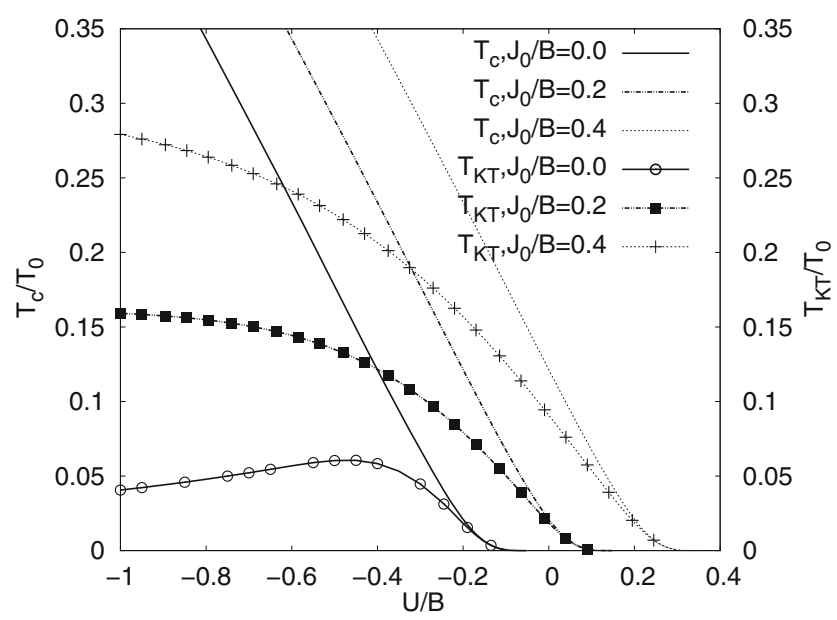

Fig. 11 Transition temperatures $T_{K T}$ and $T_{c}$ plotted as a function of $U / B$. For $n=0.75$ and fixed values of $J_{0} / B: J_{0} / B=0$; 0.2; 0.4. $T_{0}=$ $B / k_{B}$ (SQ lattice). $B=8 t, J_{0}=4 J, T_{0}=\frac{8 t}{K_{B}}$ 

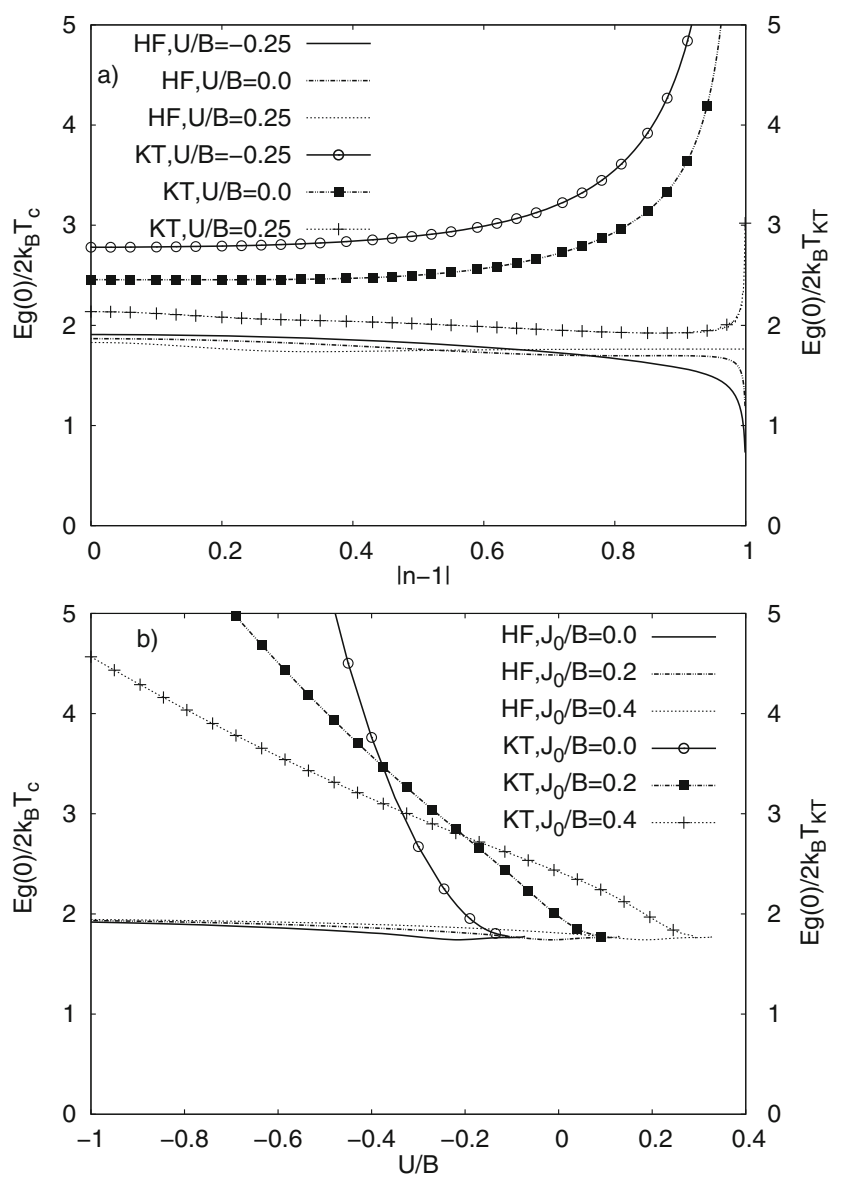

Fig. 12 The gap to critical temperatures ratios $E_{g}(0) / 2 k_{B} T_{K T / c}$ plotted (a) as a function of $-n-1-$ for $J_{0} / B=0.5$ and $U / B=0.0, U / B=0.25$, $U / B=-0.25$; (b) as a function of $U$ for $n=0.75$ and $J_{0} / B=0.0, J_{0} / B$ $=0.2, J_{0} / B=0.4$. (SQ lattice)

present the ratios $E_{g}(0) / 2 k_{B} T_{K T / c}$ vs concentration $n$ for selected values of $U / B$ and $J_{0} / B$.

Notice that for $T_{K T}$ this ratio can be much greater than for $T_{c}$. We have found, the enhancement of the ratio to be the highest at low concentrations, where it can be higher even by a factor 10 or more. Only for $\frac{J_{0}-U}{t} \rightarrow 0$ we recover the BCS ratio $E_{g}(0) / 2 k_{B} T_{c} \sim 1.76$. Let us stress that the gap ratio goes to infinity for the local pair (the strong coupling) regime if $n \rightarrow 0$, since $E_{g}(0)$ remains finite in such a case (cf. Fig. 8a) and $T_{K T / c} \rightarrow 0$.

Within the KT scenario we also derived, for SQ lattice, the Uemura-type plots for the superconducting phase of the model, and examples of the $T_{K T / c}$ vs. $1 / \lambda^{2}$ figures for controlling parameter $n$ with fixed values of $J_{0} / B$ and $U$ are shown in Figs. 13 and 14. Apart from the weak coupling regime the curves plotted on the figures have shapes analogous to the Uemura's plots experimentaly obtained $[55,57]$ for various classes of the "exotic" superconductors (the superconductors with short-coherence length) such as bismuthates, the organic materials and cuprates. For

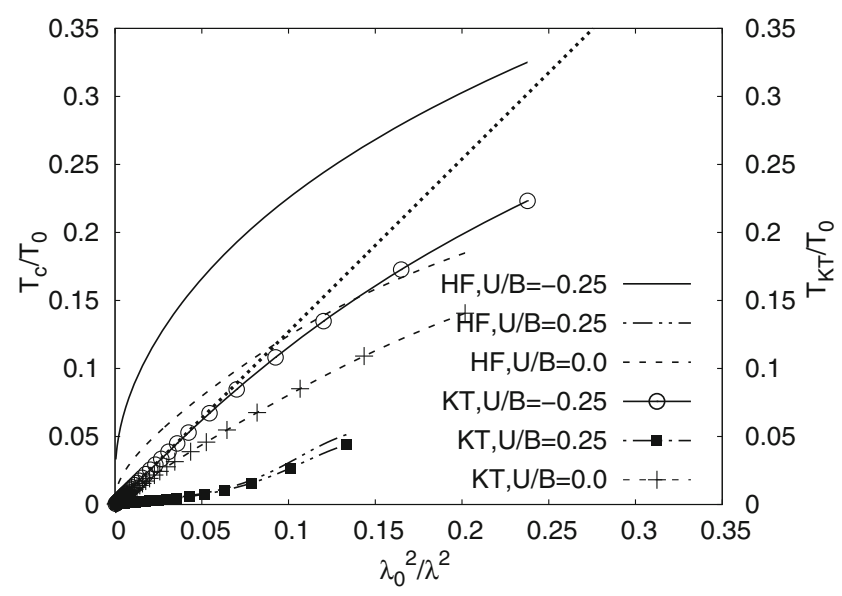

Fig. 13 The Uemura-type plots: $T_{c / p} \mathrm{vs} \lambda_{0}^{2} / \lambda^{2}$, with the controlling variable $n$, for $J_{0} / B=0.5$ and several fixed values of $U(U / B=0.0, U / B$ $=0.25, U / B=-0.25)$. The straight dotted line marks an upper bound for the phase ordering temperature $Q \rho_{s}(0),(Q \simeq 0.898$ see (26)). $\lambda_{0}^{2} / \lambda^{2}=4 \rho_{s} / B, \lambda_{0}=\frac{\hbar c}{e} \sqrt{\frac{a^{d-2}}{4 \pi \mathrm{B}}} \cdot T_{0}=B / k_{B}$ (SQ lattice).

low concentration $n$ the experimental points adhere to the universal $Q \lambda^{2}(0)$ line (the results presented in Figs. 13 and 14 were obtained for $Q \simeq 0.898$ (see (26)). It should be emphasized that $T_{c}$ is not related to $\rho_{s}$ and analogous plots with $T_{c}$ cannot account for the scaling [12, 33, 52,

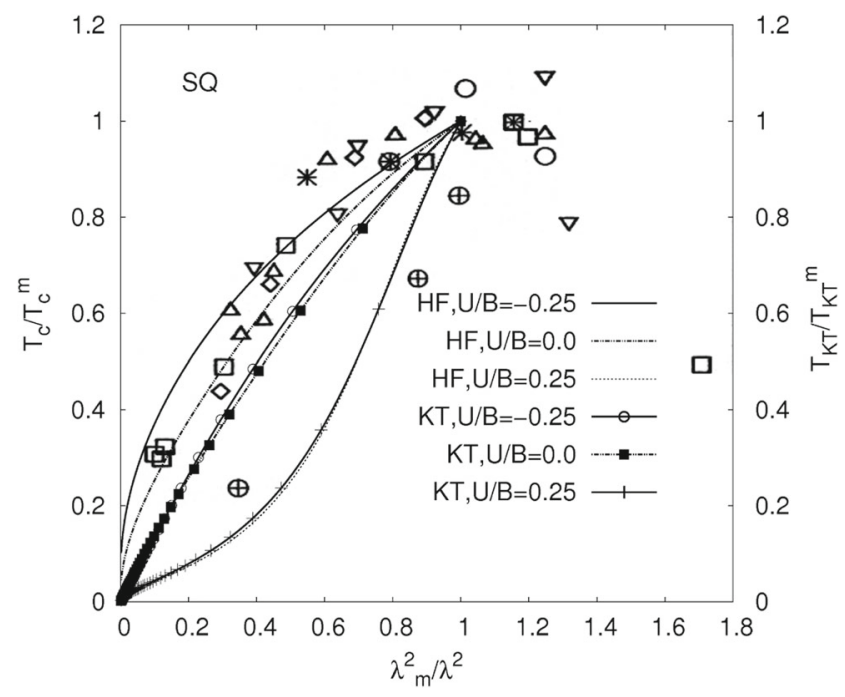

Fig. 14 The Uemura-type plot: $\frac{T_{c}}{T_{c}^{m}}$ vs. $\frac{\lambda_{m}^{2}}{\lambda^{2}}$ and $\frac{T_{K T}}{T_{K T}^{m}}$ vs. $\frac{\lambda_{m}^{2}}{\lambda^{2}}$, where $\frac{\lambda_{m}^{2}}{\lambda^{2}}$ is a ground state value. Plotted for controlling variable $n$, for $J_{0} / B=0.5$ and several fixed values of $U(U / B=0.0, U / B=0.25$, $U / B=-0.25) . \lambda_{m}$ corresponds to the relevant maximum transition temperature, $T_{c}^{m}$ or $T_{K T}^{m}$ respectively. (o)- $\mathrm{Tl}_{2} \mathrm{Ba}_{2} \mathrm{Ca}_{2} \mathrm{Cu}_{3} \mathrm{O}_{10}, \mathrm{Tl}_{0.5}$ $\mathrm{Pb}_{0.5} \mathrm{Sr}_{2} \mathrm{Ca}_{2} \mathrm{Cu}_{3} \mathrm{O}_{9}, \mathrm{Bi}_{2-\mathrm{x}} \mathrm{Pb}_{\mathrm{x}} \mathrm{Sr}_{2} \mathrm{Ca}_{2} \mathrm{Cu}_{3} \mathrm{O}_{16} ;()-\mathrm{Y}_{1-\mathrm{x}} \mathrm{Pr}_{\mathrm{x}} \mathrm{Ba}_{2}$ $\mathrm{Cu}_{3} \mathrm{O}_{6.97} ;(\triangle)-\mathrm{YBa}_{2} \mathrm{Cu}_{3} \mathrm{O}_{\mathrm{x}} ;(\nabla)-\mathrm{La}_{2-\mathrm{x}} \mathrm{Sr}_{x} \mathrm{CuO}_{4} ;(\star)-\mathrm{Bi}_{2} \mathrm{Sr}_{2} \mathrm{Ca}_{1-\mathrm{x}}$ $\mathrm{Y}_{x} \mathrm{Cu}_{2} \mathrm{O}_{8+\delta} ;(\sqcap)-\mathrm{LaMo}_{6} \mathrm{Se}_{2}, \mathrm{PbMo}_{6} \mathrm{~S}_{8}, \mathrm{SnMo}_{6} \mathrm{~S}_{4} \mathrm{Se}_{4}, \mathrm{SnMo}_{6} \mathrm{~S}_{7} \mathrm{Se}$, $\mathrm{SnMo}_{6} \mathrm{~S}_{1} \mathrm{Se}_{7}, \mathrm{LaMo}_{8} \mathrm{~S}_{8}, \mathrm{PbMo}_{6} \mathrm{~S}_{4} \mathrm{Se}_{4} ;(\oplus)-\mathrm{Tl}_{2} \mathrm{Ba}_{2} \mathrm{CuO}_{6+\delta}$ (for overdoped range). Experimental data taken from $[58,59]$ 
53]. Let us point out that for $t_{2} \neq 0$ [18] the densities of states for SQ and SC lattices are no longer symmetric and the function $T_{K T}\left(1 / \lambda^{2}\right)$ does not represent the one-valued function, whereas it exhibits substantial hysteresis due to differences in positions of the maxima values of the $T_{K T}(n)$ and $1 / \lambda^{2}(n)$.

In Fig. 14 we compared our results with experimental data corresponding mainly to SQ lattices (cuprates) and alternating cubic lattices (Chevrel phases, doped $\mathrm{BaBi}_{3}$ ) $[58,59,76,77]$. In order to compare the results we have normalized $T_{c}$ and $T_{K T}$ to unity by scaling both temperatures over respective maximal values of the temperatures: $T_{c}^{m}$ and $T_{K T}^{m}$, and $1 / \lambda^{2}$ we scaled over a value $1 / \lambda_{m}^{2}$ corresponding to the value of the respective maximal temperature. The experimental data were scaled analogously. Except for the overdoped cases the data compare best with the plots obtained for the values of coupling between strong and intermediate. The experimentally found deviations from the universal dependence in the strongly overdoped regime can be explained either by the asymmetry in $\mathcal{D}(\varepsilon)$ due to further neighbour hopping in the considered lattices or by the effects of intersite Coulomb interactions [79], which can be different for various families of materials.

In Fig. 15 we qualitatively compare the theoretical plots $\lambda(n)$ obtained for the discussed simple effective model with penetration depth measurements reported by Locquet [48] for $\mathrm{La}_{2-\mathrm{x}} \mathrm{Sr}_{\mathrm{x}} \mathrm{CuO}_{4}$ films. The experimental data extend from the regime of heavily underdoped to heavily overdoped. The theoretical results fit well the experimental measurements.

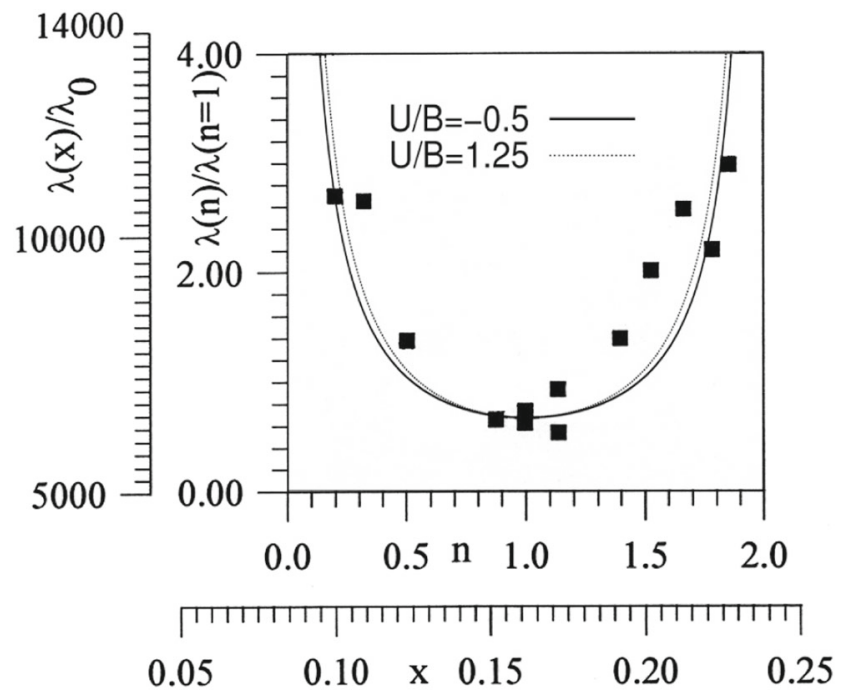

Fig. 15 The $\lambda(n) / \lambda(n=1)$ at $T=0 \mathrm{~K}$ for $J / B=0.5$ and several fixed values of $U / B$ for SQ lattice, compared with experimental results [48] obtained for $\mathrm{La}_{2-\mathrm{x}} \mathrm{Sr}_{\mathrm{x}} \mathrm{CuO}_{4}$ films (-39 nm) (full squares), $\lambda_{0}=\lambda(x$ $=0.159)=[6700 \pm 500] \AA$

\section{Conclusions and Remarks}

We have explored in this work the ground state and final temperature phase diagrams as well as the superconducting properties of the Penson-Kolb-Hubbard model. The study have been performed for arbitrary electron density and $J$ $>0$. In this model, the intersite charge exchange interaction (a non-local interaction) is responsible for pairs formation and condensation.

The PKH model can be treated as an effective model of the s-wave superconductors with short coherence length, which can be of order of interparticle distance or even a lattice constant and can be applied to isotropic $d=3$ structures (such as the barium-bismuthate compounds [80, 82], Chevrel phases [1] and fullerides [83-87]), as well as those with layered $(d=2)$ structures (such as heavy fermion systems and some organic superconductors). Moreover, the model may also lead to some qualitative understanding of the $d=2$ high $-T_{c}$ cuprates $[76,88,91]$, iron pnictides and chalcogenides [5, 93].

For the SQ lattice we have determined the stability of s-wave pairing state with respect to $\mathrm{F}, \mathrm{AF}$ and $\mathrm{N}$ phases. We have presented the ground state diagrams for homogeneous phases only (without considering PS states) and a preliminary phase diagram involving PS states (Figs. 1-3). In the diagrams the phase transitions to nonordered state are of the 2nd-order, and all the transition lines separating considered ordered states are of the 1storder. Increasing $U / 4 t$ broadened $\mathrm{F}$ phase concentrations $n$ range and reduced the $n$ ranges of $\mathrm{AF}$ and $\mathrm{N}$ phases occurrence. We have shown that at $T=0$ for $J>U / z$, in the general case of arbitrary $U / 4 t$ and $J / 4 t$ the $S$-phase was always stable with respect to the $\mathrm{N}$ phase for any value of the concentration $n$ and single electron hopping.

In the diagram involving PS states (Fig. 3) we have shown that the homogeneous ordered phases were separated by the respective PS states and homogeneous AF phase could occur only at half-filling. For $n \neq 1$ the AF ordering could appear only within the phase separated states: $\mathrm{PS}(\mathrm{S} / \mathrm{AF})$ and $\mathrm{PS}(\mathrm{AF} / \mathrm{F})$ in a limited extent of concentration $n$ near to the half-filling.

At $T=0$ the isotropic s-wave pairing, S-phase, stable for the attractive $(J>0)$ and the attractive $U(U<0)$ is found to exhibit a smooth crossover between the BCS-like regime and the tightly bound pairs limit with increasing pairing interactions. This behaviour is qualitatively different from the one observed for repulsive $J(J<0)$ when the transition into the eta-phase occurs only above a critical value $-J_{c}-$ dependent on band filling $(n)$ and lattice structure. In this case the system never manifests standard BCS-like features [16-18]. In the figures we have marked the crossover to the BEC which at $T=0$ was located after Leggett's criterion. Within homogeneous S-phase, for a given $n$, the system 
could undergo BCS-BEC crossover with decreasing $U$. The effect of the repulsive (attractive) $U$ is the reduction (expansion) of the composite bosons (BEC) regime. We have demonstrated that in a specific range of $U$ and $J$ the crossover from the weakly to strongly coupled electron pairs could be achieved by lowering the $n$. With decreasing $n$ the crossover regime is shifted towards lower values of pairing interaction. Notice that the crossover always occurs beyond the range of stability of the PS states involving s-wave domains (cf. Fig. 3). We have also presented examples of the finite temperature phase diagrams involving homogeneous states as a function of concentration and interaction $U$ (Figs. 4 and 5).

The evolution of the thermodynamic and electromagnetic superconducting properties with electron concentration and pair interactions has been analysed for $d$-dimensional hypercubic lattices (primarily for SQ lattice) and the changes in the behaviour of these characteristics at the crossover between the BCS-like limit and the local pair regime superconductivity have been discussed. We have determined the evolution of the critical temperatures $T_{c}$ (Hartree-Fock), $T_{K T}$ (Kosterlitz-Thouless) as well as the critical fields, the penetration depth, the coherence length and Ginzburg ratio at $T=0$ as a function of particle concentration $n$ and interactions.

As the pairing interactions $J_{0}$ or $-U$ increase the swave phase characteristics evolve smoothly between the regimes of the weakly interacting single particle carriers and of tightly bound pairs. The $\lambda^{-2}$ increases with increasing $J_{0} / B$ for any value of $U / B$, and the increase is linear for large $J$. This behaviour is qualitatively different from that found for the AH model $\left(J_{0}=0, U<0\right)$, where $\lambda^{-2}$ continuously decreases with increasing $-U-\mid B$ and $\lambda^{-2} \sim t^{2} /|U|$ for $-U-l B>>1$. In the strong interaction regime the electromagnetic and thermodynamic characteristics of the S-phase (for $J>0$ ) become similar to the properties of the eta-phase (for $J<0$ ). For the arbitrary coupling strengths in the low density regime $\lambda^{-2} \sim n$ as for the fermions in the continuum.

The PKH model reduces to PK model for $U=0$ and for $J=0$ reduces to AH model. The evolution of superconducting characteristics with increasing pairing interaction is essentially different in both systems. For the PK model, in the limit of strong coupling, values of $\lambda^{-2}$, $H_{c}, T_{K T}, \kappa^{-1}$ increase with $J$ while for the AH model the characteristics decrease with $|U|$.

Our results obtained for the case $U=0, J>0$ are consistent with those obtained for collective excitations performed using a generalized random-phase approximation [28, 36]. The collective mode velocity, in the PK model, rise with $J$ [28] while in the AH model it drops with $|U|$ [36]. Moreover, for the studied models, the conclusions are in qualitatively good agreement with the results of perturbational expansions both in the weak and strong interaction regimes [30, 94, 95].

The S-phase can be stable for repulsive values of $U$ $\left(0<U<U_{c}\right)$ as well. In the PKH model, repulsive $U$ modifies the S-phase characteristics (cf. also [17]) and shrinks the extent of the superconducting phase stability. It also can induce 1 st or 2 nd order transitions to various nonsuperconducting phases.

Apart from the weak coupling regime one finds a substantial increase in the gap to critical temperature ratio resulting from the phase fluctuations and the energy scales separation for the pairs formation $\left(\sim k_{B} T_{c}\right)$ and the phase coherence ( $\sim k_{B} T_{K T}$ ). Between $T_{c}$ and $T_{K T}$ a disordered (incoherent) pair state is possible in which the single-electron excitation spectrum has a gap, but the pairs are phase disordered. The $T_{K T}$ can be substantially lower than the $T_{c}$.

An important result of our work is the derivation of Uemura-type plots for the model considered. Beyond the regime of weak coupling and low concentrations the results show universal scaling: $T_{K T} \sim 1 / \lambda^{2}(0)$, similarly as it was found for the attractive Hubbard model [33], the PK model [12] and the extended Hubbard model with intersite attraction [52, 53]. Apart from the weak coupling regime, the resulting plots have a shape consistent with the experimental Uemura's plots for various classes of superconductors with short-coherence length [17]. Our results support the conclusion [12, 30, 52, 53] that in materials with short coherence length, the ground for the Uemura scaling is a separation of the energy scales of the pairs formation and the phase coherence in the underdopped limit or in the strong interaction regime.

Acknowledgements I would like to thank S. Robaszkiewicz, R. Micnas, T. Kostyrko, and K. J. Kapcia for helpful discussions.

Open Access This article is distributed under the terms of the Creative Commons Attribution 4.0 International License (http://creativecommons.org/licenses/by/4.0/), which permits unrestricted use, distribution, and reproduction in any medium, provided you give appropriate credit to the original author(s) and the source, provide a link to the Creative Commons license, and indicate if changes were made.

\section{Appendix A: Free Energies and Self-Consistent Equations for the Magnetic Phases Competing with s-wave Paring}

For arbitrary electron concentration $n$ the stable solutions are determined as the minima of free energy of the system $F_{\alpha}(\alpha=\mathrm{AF}, \mathrm{F})$ with respect to the variational parameters $x_{\alpha}$, $\mu$ and $p$, i.e. by the equations

$$
\frac{\partial F}{\partial x_{\alpha}}=0, \frac{\partial F}{\partial \mu}=0 \text { and } \frac{\partial F}{\partial p}=0
$$


from which we get sets of self-consistent equations for each considered ordering type. The order parameters for the phases considered: (i) antiferromagnetic (AF):

$x_{A F}=\frac{1}{2 N} \sum_{i, \sigma} \sigma \exp \left(\mathbf{Q}_{i} \cdot \mathbf{R}_{i}\right)\left\langle c_{i \sigma}^{+} c_{\iota \sigma}\right\rangle$,

(ii) ferromagnetic $(\mathrm{F})$ :

$x_{F}=\frac{1}{N} \sum_{i, \sigma} \sigma\left\langle c_{i \sigma}^{+} c_{l \sigma}\right\rangle$,

The equations for the phases considered derived for the alternated lattice, (i.e. $k+Q=-k$ ) in the (broken symmetry) HFA: the free energy of the ferromagnetic phase $F_{F}$ :

$$
\begin{aligned}
F_{F}= & \bar{\mu}(n-1)+\frac{4}{z} J p^{2}+\frac{1}{4} U n^{2}+U x_{F}^{2}+ \\
& -\frac{2 T}{N} \sum_{k, r} \ln \left[2 \cosh \left(\frac{E_{F}^{r}(k)}{2 T}\right)\right],
\end{aligned}
$$

the thermodynamic potential of the ferromagnetic phase $\Omega_{F}$ :

$$
\begin{aligned}
\Omega_{F}= & -\bar{\mu}+\frac{4}{z} J p^{2}-\frac{1}{4} U n^{2}+U x_{F}^{2}+ \\
& -\frac{2 T}{N} \sum_{k, r} \ln \left[2 \cosh \left(\frac{E_{F}^{r}(k)}{2 T}\right)\right],
\end{aligned}
$$

the set of self-consistent equations: (i) the equation for the number of particles of the ferromagnetic phase:

$n-1=-\frac{1}{2} \sum_{k, r} \tanh \left(\frac{E_{F}^{r}(k)}{2 T}\right)$,

(ii) the equation for the order parameter of the ferromagnetic phase:

$x_{F}=-\frac{1}{2} \sum_{k, r} r \tanh \left(\frac{E_{F}^{r}(k)}{2 T}\right)$,

where $E_{F}^{ \pm}(k)=\bar{k} \pm U x_{F}, \bar{k}={ }_{k}-\bar{\mu}, \bar{\mu}=\mu-U n / 2$.

The free energy of the antiferromagnetic phase $F_{A F}$ :

$$
\begin{aligned}
F_{A F}= & \bar{\mu}(n-1)+\frac{4}{z} J p^{2}+\frac{1}{4} U n^{2}+U x_{A F}^{2}+ \\
& -\frac{2 T}{N} \sum_{k, r} \ln \left[2 \cosh \left(\frac{E_{A F}^{r}(k)}{2 T}\right)\right],
\end{aligned}
$$

the thermodynamic potential of the antiferromagnetic phase $\Omega_{A F}$ :

$$
\begin{aligned}
\Omega_{A F}= & -\bar{\mu}+\frac{4}{z} J p^{2}-\frac{1}{4} U n^{2}+U x_{A F}^{2}+ \\
& -\frac{2 T}{N} \sum_{k, r} \ln \left[2 \cosh \left(\frac{E_{A F}^{r}(k)}{2 T}\right)\right],
\end{aligned}
$$

the set of self-consistent equations: (i) the equation for the number of particles of the antiferromagnetic phase:

$n-1=-\frac{1}{2} \sum_{k, r} \tanh \left(\frac{E_{A F}^{r}(k)}{2 T}\right)$, (ii) the equation for the order parameter of the antiferromagnetic phase:

$\frac{2}{U}=\sum_{k} \frac{\tanh \left(\frac{E_{A F}^{+}(k)}{2 T}\right)-\tanh \left(\frac{E_{A F}^{-}(k)}{2 T}\right)}{E_{A F}^{+}(k)-E_{A F}^{-}(k)}$,

and the Fock term:

$p=\frac{-1}{4 N} \sum_{k} \gamma_{k} \frac{1}{E_{\mathrm{AFk}}^{+}-E_{\mathrm{AFk}}^{-}} \tanh \left(\frac{E_{\mathrm{AFk}}^{+}+E_{\mathrm{AFk}}^{-}}{2 T}\right)$,

where $E_{A F}^{ \pm}(k)=\bar{\mu} \pm \sqrt{2+U^{2} x_{A F}^{2}}$.

Publisher's note Springer Nature remains neutral with regard to jurisdictional claims in published maps and institutional affiliations.

\section{References}

1. Micnas, R., Ranninger, J., Robaszkiewicz, S.: Rev. Mod. Phys. 62 , 113 (1990). https://doi.org/10.1103/RevModPhys.62.113

2. Robaszkiewicz, S., Pawłowski, G.: Phys. C. 210, 61 (1993). https://doi.org/10.1016/0921-4534(93)90009-F

3. Robaszkiewicz, S.: Acta Phys. Pol. A 85, 117 (1994). https://doi.org/10.12693/APhysPolA.85.117

4. Dagotto, E., Hotta, T., Moreo, A.: Phys. Rep. 344, 1 (2001). https://doi.org/10.1016/S0370-1573(00)00121-6

5. Johnston, D.C.: Adv. in Phys. 59, 803 (2010). https://doi.org/10. 1080/00018732.2010.513480

6. Kapcia, K., Robaszkiewicz, S., Micnas, R.: J. Phys.: Condens. Matter 24, 215601 (2012). https://doi.org/10.1088/0953$8984 / 24 / 21 / 215601$

7. Kapcia, K.: J. Supercond. Nov. Magn. 27, 913 (2014). https://doi.org/10.1007/s10948-013-2409-8

8. Penson, K.A., Kolb, M.: Phys. Rev. B 33, 1663 (1986). https://doi.org/10.1103/PhysRevB.33.1663

9. Japaridze, G.I., Muller-Hartmann, E.: J. Phys.: Condens. Matter 9, 10509 (1997). https://doi.org/10.1088/0953-8984/9/47/018

10. Japaridze, G.I., Kampf, A.P., Sekania, M., Kakashvili, P., Brune, Ph.: Phys. Rev. B 65, 014518 (2001). https://doi.org/10.1103/ PhysRevB.65.014518

11. Robaszkiewicz, S., Bułka, B.R.: Phys. Rev. B 59, 6430 (1999). https://doi.org/10.1103/PhysRevB.59.6430

12. Czart, W.R., Robaszkiewicz, S.: Phys. Rev. B 64, 104511 (2001). https://doi.org/10.1103/PhysRevB.64.104511

13. Robaszkiewicz, S., Czart, W.R.: Acta Phys. Pol. B 32, 3267 (2001)

14. Mierzejewski, M., Maśka, M.M.: Phys. Rev. B 69, 054502 (2004). https://doi.org/10.1103/PhysRevB.69.054502

15. Ptok, A., Maśka, M., Mierzejewski, M.: J. Phys.: Condens. Matter 21, 295601 (2009). https://doi.org/10.1088/0953-8984/21/ 29/295601

16. Czart, W.R., Robaszkiewicz, S.: Acta. Phys. Pol. A 106, 709 (2004). https://doi.org/10.12693/APhysPolA.106.709

17. Robaszkiewicz, S., Czart, W.: Phys. Status Solidi (b) 236, 416 (2003). https://doi.org/10.1002/pssb.200301693

18. Czart, W.R., Robaszkiewicz, S., Tobijaszewska, B.: Phys. Stat. Solidi (b) 244, 2327 (2007). https://doi.org/10.1002/pssb. 200674607

19. Kapcia, K., Robaszkiewicz, S.: J. Phys.: Condens. Matter 25, 065603 (2013). https://doi.org/10.1088/0953-8984/25/6/065603 
20. Kapcia, K.J.: Acta Phys. Pol. A 126, A-53 (2014). https://doi. org/10.12693/APhysPolA.126.A-53

21. Ptok, A., Kapcia, K.J.: Supercond. Sci. Technol. 28, 045022 (2015). https://doi.org/10.1088/0953-2048/28/4/045022

22. Kapcia, K.J., Czart, W.R., Ptok, A.: J. Phys. Soc. Jpn. 85, 044708 (2016). https://doi.org/10.7566/JPSJ.85.044708

23. Kapcia, K.J., Czart, W.R.: Acta Phys. Pol. A 130, 617 (2016). https://doi.org/10.12693/APhysPolA.130.617

24. Kapcia, K.J., Czart, W.R.: Acta Phys. Pol. A 133, 401 (2018). https://doi.org/10.12693/APhysPolA.133.401

25. Sikkema, A.E., Affleck, I.: Phys. Rev. B 52, 10207 (1995). https://doi.org/10.1103/PhysRevB.52.10207

26. van den Bossche, M., Caffarel, M.: Phys. Rev. B 54, 17414 (1996). https://doi.org/10.1103/PhysRevB.54.17414

27. Bouzerar, G., Japaridze, G.I.: Z. Phys. B 104, 215 (1997). https://doi.org/10.1007/s002570050442

28. Roy, G.K., Bhattacharyya, B.: Phys. Rev. B 55, 15506 (1997). https://doi.org/10.1103/PhysRevB.55.15506

29. Dolcini, F., Montorsi, A.: Phys. Rev. B 62, 2315 (2000). https://doi.org/10.1103/PhysRevB.62.2315

30. Czart, W., Kostyrko, T., Robaszkiewicz, S.: Physica C 272, 51 (1996). https://doi.org/10.1016/S0921-4534(96)00554-0

31. Denteneer, P.J.H., An, G., van Leeuwen, J.M.J.: Phys. Rev. B 47, 6256 (1993). https://doi.org/10.1103/PhysRevB.47.6256

32. van Leeuwen, M.J., du Crao de Jongh, M.S.L., Denteneer, P.J.H.: J. Phys. A 29, 41 (1996). https://doi.org/10.1088/03054470/29/1/008

33. Singer, J.M., Schneider, T., Pedersen, M.: Eur. Phys. J. B 2, 17 (1998). https://doi.org/10.1007/s100510050221

34. Bak, M., Micnas, R.: J. Phys.: Condens. Matter 10, 9029 (1998). https://doi.org/10.1088/0953-8984/10/40/009

35. Hui, A., Doniach, S.: Phys. Rev. B 48, 2063 (1993). https://doi.org/10.1103/PhysRevB.48.2063

36. Bhattacharyya, B., Roy, G.K.: J. Phys. Condens. Matter 7, 5537 (1995). https://doi.org/10.1088/0953-8984/7/28/011

37. Dupuis, N.: Phys. Rev. B 70, 134502 (2004). https://doi.org/10. 1103/PhysRevB.70.134502

38. Borejsza, K., Dupuis, N.: Phys. Rev. B 69, 085119 (2004). https://doi.org/10.1103/PhysRevB.69.085119

39. Japaridze, G.I., Kampf, A.P., Sekania, M., Kakashvili, P., Brune, Ph.: Phys. Rev. B 65, 014518 (2001). https://doi.org/10.1103/ PhysRevB.65.014518

40. Czart, W.R., Robaszkiewicz, S.: Acta Phys. Pol. A 97, 217 (2000). https://doi.org/10.12693/APhysPolA.97.217

41. Czart, W., Robaszkiewicz, S.: Acta Phys. Pol. A 100, 885 (2001). https://doi.org/10.12693/APhysPolA.100.885

42. Czart, W., Robaszkiewicz, S.: Acta Phys. Pol. A 127, 278 (2015). https://doi.org/10.12693/APhysPolA.127.278

43. Czart, W., Robaszkiewicz, S.: Acta Phys. Pol. A 127, 275 (2015). https://doi.org/10.12693/APhysPolA.127.275

44. Kapcia, K.: Acta Phys. Pol. A 121, 733 (2012). https://doi.org/10. 12693/APhysPolA.121.733

45. Kapcia, K.: J. Supercond. Nov. Magn. 26, 2647 (2013). https://doi. org/10.1007/s10948-013-2152-1

46. Kapcia, K.J.: Acta Phys. Pol. A 127, 204 (2015). https://doi. org/10.12693/APhysPolA.127.204

47. Kapcia, K.J.: J. Supercond. Nov. Magn. 28, 1289 (2015). https://doi.org/10.1007/s10948-014-2906-4

48. Locquet, J.P., Jaccard, Y., Cretton, A., Williams, E.J., Arrouy, F., Mächler, E., Schneider, T., Fischer, O., Martinoli, P.: Phys. Rev. B 54, 7481 (1996). https://doi.org/10.1103/PhysRevB.54.7481

49. Schneider, T.: Polarons and Bipolarons in High $T_{c}$ Superconductors and Related Materials. In: Salje, E.K.H., Alexandrov, A.S., Liang, W.Y. (eds.) Cambridge University Press. https://doi.org/10.1017/CBO9780511599811, p 258 (1995)
50. Chattopadhyay, B.: Phys. Lett. A 226, 231 (1997)

51. Chattopadhyay, B., Gaitonde, D.M., Taraphder, A.: Europhys. Lett. 34, 705 (1996). https://doi.org/10.1209/epl/i1996-00518-y

52. Micnas, R., Robaszkiewicz, S., Tobijaszewska, B.: J. Supercond. 12, 79 (1999). https://doi.org/10.1023/A:1007729821661

53. Tobijaszewska, B., Micnas, R.: Acta Phys. Pol. A 97, 393 (2000). https://doi.org/10.12693/APhysPolA.97.393

54. Micnas, R., Tobijaszewska, B.: J. Phys. Condens. Matter 14, 9631 (2002). https://doi.org/10.1088/0953-8984/14/41/319

55. Uemura, Y.J., Luke, G.M., Sternlieb, B.J., Brewer, J.H., Carolan, J.F., Hardy, W.N., Kadono, W.N., Kempton, J.R., Kiefl, R.F., Kreitzman, S.R., Mulhern, P., Riseman, T.M., Williams, D.Ll., Yang, B.X., Uchida, S., Takagi, H., Gopalakrishnan, J., Sleight, A.W., Subramanian, M.A., Chien, C.L., Cieplak, M.Z., Xiao, G., Lee, V.Y., Statt, B.W., Stronach, C.E., Kossler, W.J., Yu, X.H.: Phys. Rev. Lett. 62, 2317 (1989). https://doi.org/10.1103/PhysRevLett. 62.2317

56. Uemura, Y.J., Le, L.P., Luke, G.M., Sternlieb, B.J., Wu, W.D., Brewer, J.H., Riseman, T.M., Seaman, C.L., Maple, M.B., Ishikawa, M., Hinks, D.G., Jorgensen, J.D., Saito, G., Yamochi, H.: Phys. Rev. Lett. 66, 2665 (1991)

57. Uemura, Y.J.: Physica C 282, 194 (1997). https://doi.org/10, 1016/S0921-4534(97)00194-9

58. Niedermayer, Ch., Bernhard, C., Binninger, U., Glückler, H., Tallon, J.L., Ansaldo, E.J., Budnick, J.I.: Phys. Rev. Lett. 71, 1764 (1993). https://doi.org/10.1103/PhysRevLett.71.1764

59. Uemura, Y.J., et al.: Nature 352, 605 (1991). https://doi.org/10. $1038 / 352605 \mathrm{a} 0$

60. Scalapino, D.J., White, S.R., Zhang, S.: Phys. Rev. B 47, 7995 (1993). https://doi.org/10.1103/PhysRevB.47.7995

61. Kostyrko, T., Micnas, R., Chao, K.A.: Phys. Rev. B 49, 6158 (1994)

62. Czart, W., Szkudlarek, M., Robaszkiewicz, S.: Mol. Phys. Rep. 15/16, 163 (1996)

63. Czart, W., Szkudlarek, M., Robaszkiewicz, S.: Acta Phys. Pol. A 91, 415 (1997). https://doi.org/10.12693/APhysPolA.91.415

64. Jarrell, M.: Phys. Rev. Lett. 69, 168 (1992). https://doi.org/10. 1103/PhysRevLett.69.168

65. Fradkin, E., Hirsch, J.E.: Phys. Rev. B 27, 1680 (1983). https://doi.org/10.1103/PhysRevB.27.1680

66. Miyake, K., Matsuura, T., Jichu, H., Nagaoka, Y.: Prog. Theor. Phys. 72, 1063 (1984). https://doi.org/10.1143/PTP.72.1063

67. Robaszkiewicz, S., Micnas, R., Ranninger, J.: Phys. Rev. B 36, 180 (1987). https://doi.org/10.1103/PhysRevB.36.180

68. Bastide, C., Lacroix, C.: J. Phys. C 21, 3557 (1988). https://doi.org/10.1088/0022-3719/21/19/009

69. Rosch, A., Rasch, D., Binz, B., Vojta, M.: Phys. Rev. Lett. 101, 265301 (2008). https://doi.org/10.1103/PhysRevLett.101.265301

70. Ptok, A., Crivelli, D., Kapcia, K.J.: Supercond. Sci. Technol. 28, 045010 (2015). https://doi.org/10.1088/0953-2048/28/4/045010

71. Fetter, A.L., Walecka, J.D.: Quantum Theory of Many-Particle Systems. McGraw-Hill, New York (1971)

72. Gupta, R., DeLapp, J., Batrouni, G.G., Fox, G.C., Baillie, C.F., Apostolakis, J.: Phys. Rev. Lett. 61, 1996 (1988). https://doi.org/10.1103/PhysRevLett.61.1996

73. Kosterlitz, J.M., Thouless, D.J.: J. Phys. C 6, 1181 (1973). https://doi.org/10.1088/0022-3719/6/7/010

74. Leggett, A.J.: In: Pekalski, A., Przystawa, J. (eds.) Modern Trends in the Theory of Condensed Matter. https://doi.org/10.1007/ BFb0120123, p 13. Springer, Berlin (1980)

75. Jelitto, R.J.: J. Phys. Chem. Solids 30, 609 (1969). https://doi. org/10.1016/0022-3697(69)90016-X

76. Schneider, T., Beck, H., Bormann, D., Meintrup, T., Schafroth, S., Schmidt, A.: Physica C 216, 432 (1993). https://doi.org/10.1016/ 0921-4534(93)90085-5 
77. Schneider, T., Keller, H.: Phys. Rev. Lett. 69, 3374 (1992). https://doi.org/10.1103/PhysRevLett.69.3374

78. Schneider, T., Keller, H.: Int. J. Mod. Phys. B 8, 487 (1994). https://doi.org/10.1142/S021797929400021X

79. Robaszkiewicz, S., Micnas, R., Kostyrko, T.: Physica C 235-240, 1827 (1994). https://doi.org/10.1016/0921-4534(94)92135-0

80. Taraphder, A., et al.: Phys. Rev. B 52, 1368 (1995). https://doi. org/10.1103/PhysRevB.52.1368

81. Taraphder, A., et al.: Europhys. Lett. 21, 79 (1993). https://doi. org/10.1209/0295-5075/21/1/014

82. Varma, C.M.: Phys. Rev. Lett. 61, 2713 (1989). https://doi.org/10. 1103/PhysRevLett.61.2713

83. Sarker, S.K.: Phys. Rev. B 49, 12047 (1994). https://doi.org/10. 1103/PhysRevB.49.12047

84. Chakravarty, S., Kivelson, S.: Europhys. Lett. 16, 751 (1991). https://doi.org/10.1209/0295-5075/16/8/008

85. Chakravarty, S., Gelfand, M.P., Kivelson, S.: Science 254, 970 (1991). https://doi.org/10.1126/science.254.5034.970

86. Wilson, J.A.: Physica C 182, 1 (1991). https://doi.org/10.1016/ 0921-4534(91)90448-8
87. Zhang, F.C., Ogata, M., Rice, T.M.: Phys. Rev. Lett. 67, 3452 (1991). https://doi.org/10.1103/PhysRevLett.67.3452

88. Wilson, J.A.: J. Phys. C: Solid State Phys. 20, L 911 (1987). https://doi.org/10.1088/0022-3719/20/32/007

89. Wilson, J.A.: J. Phys. C: Solid State Phys. 21, 2067 (1988). https://doi.org/10.1088/0022-3719/21/11/003

90. Wilson, J.A.: Physica C 233, 332 (1994). https://doi.org/10.1016/ 0921-4534(94)90760-9

91. Mott, N.F.: Physica A 200, 127 (1993). https://doi.org/10.1016/ 0378-4371(93)90511-2

92. Mott, N.F.: J. Phys. Condens. Matt. 5, 3487 (1993). https://doi. org/10.1088/0953-8984/5/22/003

93. Dagotto, E.: Rev. Mod. Phys. 85, 849 (2013). https://doi.org/10. 1103/RevModPhys.85.849

94. Bułka, B., Robaszkiewicz, S.: Phys. Rev. B 54, 13138 (1996). https://doi.org/10.1103/PhysRevB.54.13138

95. van Dongen, P.G.J.: Phys. Rev. Lett. 67, 757 (1991). https://doi. org/10.1103/PhysRevLett.67.757

96. van Dongen, P.G.J.: Phys. Rev. B 50, 14016 (1994). https://doi. org/10.1103/PhysRevB.50.14016 